\title{
Shell model of turbulence perturbed by Lévy noise
}

\author{
Utpal Manna and Manil T. Mohan
}

\begin{abstract}
In this work we prove the existence and uniqueness of the strong solution of the shell model of turbulence perturbed by Lévy noise. The local monotonicity arguments have been exploited in the proofs.
\end{abstract}

Mathematics Subject Classification (2000). Primary 60H15;

Secondary 76D03, 76D06.

Keywords. GOY model, Lévy processes, Local monotonicity.

\section{Introduction}

Stochastic partial differential equations driven by jump processes gain attention quiet recently due to its important applications in Mathematical Physics (see $[4,29]$ ) and also in Biomathematics (see [32]). Detailed literature on this subject can be found in the books by Applebaum [5], Ikeda and Watanabe [15], and Peszat and Zabczyk [26] (and references therein). In the last few years several interesting results have been established. To name a few, de Acosta [1,2] first studied the large deviations for Lévy processes on Banach spaces and large deviations for solutions of stochastic differential equations driven by Poisson measures; Albeverio et al. [3] proved the existence and uniqueness for solutions of parabolic SPDEs driven by Poisson random measures; absolute continuity of the law of the solutions of parabolic SPDEs driven by Poisson random measures was proved by Fournier [11] using techniques from Malliavin calculus; Hausenblas [13], Mandrekar and Rüdiger [20] and Rüdiger [28] extensively studied the existence and uniqueness of stochastic integral equations driven by Lévy noise and compensated Poisson random measures on separable Banach spaces; Mueller [24] proved the short time existence for the solutions (which is a minimal solution) of stochastic heat equation with non-negative Lévy noise; Röckner and Zhang [27] established the existence and uniqueness results for solutions of stochastic evolution equations driven by Lévy noise and obtained the large deviation principles for the additive Lévy noise case; Zhao and Chao [33] established the global existence and uniqueness of the strong solution for 2D Navier-Stokes equations on the torus perturbed by a Lévy process. 
This work deals with an infinite dimensional shell model, a mathematical turbulence model that received increasing attention in recent years. Apparently there are only a few rigorous works on infinite dimensional shell model, namely $[6,9]$ one in the deterministic case and the other in the stochastic case with additive noise respectively. In both of these works a variational semigroup formulation has been introduced. In [21] the existence and uniqueness of the strong solutions of the stochastic shell model of turbulence perturbed by multiplicative noise have been proved. The authors have also established a large deviation principle for the solution of the shell model. Our present work deals with a more general stochastic model, with Lévy noise: the proofs of existence and uniqueness of strong solutions are considerably more difficult in this case.

In this paper, in the framework of Gelfand triple $V \subset H \cong H^{\prime} \subset V^{\prime}$ (see Sect. 3 for precise definitions), we consider the following abstract form of the GOY model of turbulence with Lévy noise:

$$
\begin{aligned}
& \mathrm{d} u+[\nu A u+B(u, u)] \mathrm{d} t=f(t) \mathrm{d} t+\sqrt{\varepsilon} \sigma(t, u) \mathrm{d} W(t)+\varepsilon \int_{Z} g(u, z) \tilde{N}(d t, d z) \\
& u(0)=u_{0},
\end{aligned}
$$

The operators $A$ and $B$ are defined in Sect. 3. $W(t)$ is an $H$-valued Wiener process with positive symmetric trace class covariance operator $Q \cdot \tilde{N}(d t, d z)=$ $N(d t, d z)-d t \lambda(d z)$ is a compensated Poisson random measure (cPrm), where $N(d t, d z)$ denotes the Poisson counting measure associated to Poisson point process $p(t)$ on $Z$ and $\lambda(d z)$ is a $\sigma$-finite measure on $(Z, \mathcal{B}(Z))$.

The main result of the paper is the following theorem. The spaces $V, V^{\prime}, H, H_{0}, L_{Q}\left(H_{0} ; H\right), \mathbb{H}_{\lambda}^{2}([0, T] \times Z ; H), \mathcal{D}([0, T] ; H)$ which appear in the statement of this theorem are defined in Sect. 2.

Theorem 1.1. (Main Theorem) Let us consider the above stochastic GOY model of turbulence driven by Lévy processes with the initial condition $u_{0}(x)$. Let $u_{0}$ be $\mathcal{F}_{0}$ measurable and $E\left|u_{0}\right|^{2}<\infty$. Let $f \in L^{2}\left(0, T ; V^{\prime}\right)$. Assume that $\sigma$ and $g$ satisfy the following hypotheses of joint continuity, Lipschitz condition and linear growth:

(i) The function $\sigma \in C\left([0, T] \times V ; L_{Q}\left(H_{0} ; H\right)\right)$, and $g \in \mathbb{H}_{\lambda}^{2}([0, T] \times Z ; H)$.

(ii) For all $t \in(0, T)$, there exists a positive constant $K$ such that for all $u \in H$,

$$
|\sigma(t, u)|_{L_{Q}}^{2}+\int_{Z}|g(u, z)|_{H}^{2} \lambda(d z) \leq K\left(1+|u|^{2}\right) .
$$

(iii) For all $t \in(0, T)$, there exists a positive constant $L$ such that for all $u, v \in H$,

$$
|\sigma(t, u)-\sigma(t, v)|_{L_{Q}}^{2}+\int_{Z}|g(u, z)-g(v, z)|_{H}^{2} \lambda(d z) \leq L|u-v|^{2} .
$$

Then there exist a unique adapted process $u(t, x, \omega)$ with regularity

$$
u \in L^{2}\left(\Omega ; L^{2}(0, T ; V) \cap \mathcal{D}(0, T ; H)\right)
$$

satisfying the above stochastic GOY model in the weak sense. 
The construction of the paper is as follows. In the next Section, we give definitions, basic properties and Itô's formula for the Lévy noise. In Sect. 3, we describe the functional setting and formulate the abstract stochastic shell model (namely GOY model) when the noise coefficients are small. In Sect. 4, we prove certain a-priori energy estimates with exponential weight. These estimates together with the local monotonicity property of the sum of the linear and non linear operators play a fundamental role to prove the existence and uniqueness of the strong solution. The main result of this paper as given in the above theorem has been proved in Sect. 4 .

\section{Preliminaries}

In this section definitions and basic properties of Hilbert space valued Wiener processes and Lévy processes have been presented. Most of the materials in this section have been borrowed from the books by Da Prato and Zabczyk [10], Applebaum [5], and Peszat and Zabczyk [26]. Interested readers may look into these books for extensive study on the subject.

Definition 2.1. Let $H$ be a Hilbert space. A stochastic process $\{W(t)\}_{0 \leq t \leq T}$ is said to be an $H$-valued $\mathcal{F}_{t}$-adapted Wiener process with covariance operator $Q$ if

(i) For each non-zero $h \in H,\left|Q^{1 / 2} h\right|^{-1}(W(t), h)$ is a standard onedimensional Wiener process,

(ii) For any $h \in H,(W(t), h)$ is a martingale adapted to $\mathcal{F}_{t}$.

If $W$ is a an $H$-valued Wiener process with covariance operator $Q$ with $\operatorname{Tr} Q<\infty$, then $W$ is a Gaussian process on $H$ and

$$
E(W(t))=0, \quad \operatorname{Cov}(W(t))=t Q, \quad t \geq 0 .
$$

Let $H_{0}=Q^{1 / 2} H$. Then $H_{0}$ is a Hilbert space equipped with the inner product $(\cdot, \cdot)_{0}$

$$
(u, v)_{0}=\left(Q^{-1 / 2} u, Q^{-1 / 2} v\right), \quad \forall u, v \in H_{0},
$$

where $Q^{-1 / 2}$ is the pseudo-inverse of $Q^{1 / 2}$. Since $Q$ is a trace class operator, the imbedding of $H_{0}$ in $H$ is Hilbert-Schmidt.

Let $L_{Q}$ denote the space of linear operators $S$ such that $S Q^{1 / 2}$ is a Hilbert-Schmidt operator from $H$ to $H$. Define the norm on the space $\mathrm{L}_{Q}$ by $|S|_{\mathrm{L}_{Q}}^{2}=\operatorname{Tr}\left(S Q S^{*}\right)$.

Definition 2.2. Let $I=[a, b]$ be an interval in $\mathbb{R}^{+}$. A mapping $g: I \rightarrow \mathbb{R}^{d}$ is said to be càdlàg if, for all $t \in[a, b], g$ has a left limit at $t$ and $g$ is right continuous at $t$, i.e.,

(i) for all sequences $\left(t_{n}, n \in \mathbb{N}\right)$ in $(a, b)$ with each $t_{n}<t$ and $\lim _{n \rightarrow \infty} t_{n}=t$ we have that $\lim _{n \rightarrow \infty} g\left(t_{n}\right)$ exists;

(ii) for all sequences $\left(t_{n}, n \in \mathbb{N}\right)$ in $(a, b)$ with each $t_{n} \geq t$ and $\lim _{n \rightarrow \infty} t_{n}=t$ we have that $\lim _{n \rightarrow \infty} g\left(t_{n}\right)=g(t)$; 
(iii) for the end-points we stipulate that $g$ is right continuous at $a$ and has left limit at $b$.

Definition 2.3. Let $\left(\Omega, \mathcal{F}, \mathcal{F}_{t}, P\right)$ be a filtered probability space, and $E$ be a Banach space. A process $\left(X_{t}\right)_{t \geq 0}$ with state space $(E, \mathcal{B}(E))$ is called a Lévy process if

(i) $\left(X_{t}\right)_{t \geq 0}$ is adapted to $\left(\mathcal{F}_{t}\right)_{t \geq 0}$,

(ii) $X_{0}=0$ a.s.,

(iii) $\left(X_{t}\right)_{t \geq 0}$ has increments of the past, i.e. $X_{t}-X_{s}$ is independent of $\mathcal{F}_{s}$ if $0 \leq s<t$

(iv) $\left(X_{t}\right)_{t \geq 0}$ is stochastically continuous, i.e. $\forall \varepsilon>0, \lim _{s \rightarrow t} P\left(\left|X_{s}-X_{t}\right|>\right.$ $\varepsilon)=0$

(v) $\left(X_{t}\right)_{t \geq 0}$ is càdlàg,

(vi) $\quad\left(X_{t}\right)_{t \geq 0}$ has stationary increments, i.e. $X_{t}-X_{s}$ has the same distribution as $X_{t-s}, 0 \leq s<t$.

The jump of $X_{t}$ at $t \geq 0$ is given by $\triangle X_{t}=X_{t}-X_{t-}$. Let $Z \in \mathcal{B}\left(\mathbb{R}^{+} \times E\right)$. We define

$$
N(t, Z)=N(t, Z, \omega)=\sum_{s: 0<s \leq t} \chi_{Z}\left(\triangle X_{s}\right)
$$

In other words, $N(t, Z)$ is the number of jumps of size $\triangle X_{s} \in Z$ which occur before or at time $t . N(t, Z)$ is called the Poisson random measure (or jump measure) of $\left(X_{t}\right)_{t \geq 0}$. The differential form of this measure is written as $N(d t, d z)(\omega)$.

We call $\tilde{N}(d t, d z)=N(d t, d z)-d t \lambda(d z)$ a compensated Poisson random measure (cPrm), where $d t \lambda(d z)$ is known as compensator of the Lévy process $\left(X_{t}\right)_{t \geq 0}$. Here $d t$ denotes the Lesbegue measure on $\mathcal{B}\left(\mathbb{R}^{+}\right)$, and $\lambda(d z)$ is a $\sigma$-finite measure on $(Z, \mathcal{B}(Z))$.

Lemma 2.4. If $X=\left(X_{t}\right)_{t \geq 0}$ is a Lévy process, then $X_{t}$ is infinitely divisible for each $t \geq 0$

For proof see Proposition 1.3.1 of [5].

Lemma 2.5. If $X=\left(X_{t}\right)_{t \geq 0}$ is a Lévy process, then

$$
\phi_{X_{t}}(u)=e^{t \eta(u)},
$$

for each $u \in \mathbb{R}^{d}, t \geq 0$, where $\eta$ is the Lévy symbol of $X_{1}$.

For proof see Theorem 1.3.3 of [5].

Lemma 2.6. If $X=\left(X_{t}\right)_{t \geq 0}$ is stochastically continuous, then the map $t \rightarrow$ $\phi_{X_{t}}(u)$ is continuous for each $u \in \mathbb{R}^{d}$.

For proof see Lemma 1.3.2 of [5].

Lemma 2.7. If $X=\left(X_{t}\right)_{t \geq 0}$ is a stochastic process and there exists a sequence of Lévy processes $\left(X_{n}, n \in \mathbb{N}\right)$ with each $X_{n}=\left(X_{n_{t}}, t \geq 0\right)$ such that $X_{n_{t}}$ converges in probability to $X_{t}$ for each $t \geq 0$ and

$$
\lim _{n \rightarrow \infty} \limsup _{t \rightarrow 0} P\left(\left|X_{n_{t}}-X_{t}\right|>a\right)=0
$$


for all $a>0$, then $X$ is a Lévy process.

For proof see Theorem 1.3.7 of [5].

Example 2.8. (Brownian motion) A (standard) Brownian motion in $\mathbb{R}^{d}$ is a Lévy process $B=\left(B_{t}\right)_{t \geq 0}$ for which

(B1) $B_{t} \sim N(0, t I)$ for each $t \geq 0$,

(B2) B has continuous sample paths.

It follows immediately from $(B 1)$ that if $\mathrm{B}$ is a standard Brownian motion then its characterestic function is given by

$$
\phi_{B_{t}}(u)=\exp \left(-\frac{1}{2} t|u|^{2}\right)
$$

for each $u \in \mathbb{R}^{d}, t \geq 0$.

Example 2.9. (The Poisson Process)The Poisson process of intensity $\lambda>0$ is a Lévy process $N$ taking values in $\mathbb{N} \cup\{0\}$ wherein each $N(t) \sim \pi(\lambda t)$, so that we have

$$
P(N(t)=n)=\frac{(\lambda t)^{n}}{n !} e^{-\lambda t}
$$

for each $n=0,1,2, \ldots$

The compensated Poisson Process $\tilde{N}=(\tilde{N}(t), t \geq 0)$ where each $\tilde{N}(t)=$ $N(t)-\lambda t$. Note that $\mathbb{E}(\tilde{N}(t))=0$. and $\mathbb{E}\left(\tilde{N}(t)^{2}\right)=\lambda t$ for each $t \geq 0$.

Lemma 2.10. Let $Z$ be bounded below, then $N(t, Z)<\infty$ (a.s) for all $t \geq 0$.

For proof see Lemma 2.3.4 of [5].

Lemma 2.11. (i) If $Z$ is bounded below, then $(N(t, Z), t \geq 0)$ is a Poisson process with intensity $\lambda(Z)$.

(ii) If $Z_{1}, \ldots, Z_{m} \in \mathcal{B}\left(\mathbb{R}^{d}-\{0\}\right)$ are disjoint and bounded below and if $s_{1}, \ldots, s_{m} \in \mathbb{R}^{+}$are distinct, then the random variables $N\left(s_{1}, Z_{1}\right), \ldots$, $N\left(s_{m}, A_{m}\right)$ are independent.

For proof see Theorem 2.3.5 of [5].

Lemma 2.12. Every Lévy process is a semimartingale.

For proof see Proposition 2.7.1 of [5].

Definition 2.13. (Poisson integration) Let $N$ be the Poisson measure associated to a Lévy process $X=\left(X_{t}\right)_{t \geq 0}$. Let $g$ be a measurable function from $\mathbb{R}^{d}$ into $\mathbb{R}^{d}$ and let $Z$ be bounded below; then for each $t>0, \omega \in \Omega$, we may define the Poisson integral of $g$ as random finite sum by

$$
\int_{Z} g(z) N(t, d z)(\omega)=\sum_{z \in Z} g(z) N(t,\{z\})(\omega)
$$

Note that each $\int_{Z} g(z) N(t, d z)$ is an $\mathbb{R}^{d}$ valued random variable and gives rise to a càdlàg stochastic process as we vary t. 
Now, since $N(t,\{x\}) \neq 0 \Leftrightarrow \Delta X_{u}=x$ for at least one $0 \leq u \leq t$, we have

$$
\int_{Z} g(z) N(t, d z)=\sum_{0 \leq u \leq t} g\left(\Delta X_{u}\right) \chi_{Z}\left(\Delta X_{u}\right) .
$$

Lemma 2.14. (The Lévy-Itô decomposition) If $X=\left(X_{t}\right)_{t \geq 0}$ is a Lévy process, then there exists $b \in \mathbb{R}^{d}$, a Brownian motion $B_{A}$ with covariance matrix $A$ and an independent Poisson random measure $N$ on $\mathbb{R}^{+} \times\left(\mathbb{R}^{d}-\{0\}\right)$ such that, for each $t \geq 0$,

$$
X_{t}=b t+B_{A}(t)+\int_{|z|<1} z \tilde{N}(t, d z)+\int_{|z| \geq 1} z N(t, d z) .
$$

For proof see Theorem 2.4.16 of [5].

Definition 2.15. Let $E$ and $F$ be separable Banach spaces. Let $F_{t}:=\mathcal{B}\left(\mathbb{R}^{+} \times\right.$ $E) \otimes \mathcal{F}_{t}$ be the product $\sigma$-algebra generated by the semi-ring $\mathcal{B}\left(\mathbb{R}^{+} \times E\right) \times \mathcal{F}_{t}$ of the product sets $Z \times F, Z \in \mathcal{B}\left(\mathbb{R}^{+} \times E\right), F \in \mathcal{F}_{t}$ (where $\mathcal{F}_{t}$ is the filtration of the additive process $\left.\left(X_{t}\right)_{t \geq 0}\right)$. Let $T>0$, and

$$
\begin{aligned}
\mathbb{H}(Z)= & \left\{g: \mathbb{R}^{+} \times Z \times \Omega \rightarrow F, \text { such that } g \text { is } F_{T} / \mathcal{B}(F)\right. \\
& \text { measurable and } \left.g(t, z, \omega) \text { is } \mathcal{F}_{t^{-}} \text {adapted } \forall z \in Z, \forall t \in(0, T]\right\}
\end{aligned}
$$

Let $p \geq 1$,

$$
\mathbb{H}_{\lambda}^{p}([0, T] \times Z ; F)=\left\{g \in \mathbb{H}(Z): \int_{0}^{T} \int_{Z} \mathbb{E}\left[\|g(t, z, \omega)\|_{F}^{p}\right] \lambda(d z) d t<\infty\right\}
$$

Let $H$ be a vector with components $\left(H^{1}, H^{2}, \ldots, H^{d}\right)$ taking values in $\mathbb{H}_{\lambda}^{2}([0, T] \times Z ; E)$; then we may construct an $\mathbb{R}^{d}$-valued process $A=(A(t), t \geq$ $0)$ with components $\left(A^{1}, A^{2}, \ldots, A^{d}\right)$ where each

$$
A^{i}(T)=\int_{0}^{T} \int_{|z| \leq 1} H^{i}(t, z) \tilde{N}(d t, d z) .
$$

The construction of $A$ extends to the case where $H$ is no longer lies in $\mathbb{H}_{\lambda}^{2}([0, T] \times Z ; E)$ but satisfies

$$
P\left(\int_{0}^{T} \int_{E}|H(t, z)| \lambda(d z) d t<\infty\right)=1 .
$$

In this case $A$ is still a local martingale. It is an $L^{1}$-martingale if

$$
\int_{0}^{T} \int_{E} \mathbb{E}(|H(t, z)|) \lambda(d z) d t<\infty .
$$

Let us introduce the compound Poisson process $P=\left(P_{t}, t \geq 0\right)$, where each $P(t)=\int_{Z} z N(t, d z)$. Let $\mathrm{K}$ be a predictable mapping; then, generalizing equation (1.1), we define

$$
\int_{0}^{T} \int_{Z} K(t, z) N(d t, d z)=\sum_{0 \leq u \leq t} K\left(u, \Delta P_{u}\right) \chi_{Z}\left(\Delta P_{u}\right)
$$


as a random finite sum.

In particular, if $H$ satisfies the square-integrability (or integrability) condition given above we may then define, for each $1 \leq i \leq d$,

$$
\int_{0}^{T} \int_{Z} H^{i}(t, z) \tilde{N}(d t, d z)=\int_{0}^{T} \int_{Z} H^{i}(t, z) N(d t, d z)-\int_{0}^{T} \int_{Z} H^{i}(t, z) \lambda(d z) d t .
$$

Definition 2.16. An $\mathbb{R}^{d}$-valued stochastic process $Y=\left(Y_{t}\right)_{t \geq 0}$ is a Lévy-type stochastic integral if it can be written in the following form, for each $1 \leq i \leq d$, $t \geq 0,1 \leq i \leq d, 1 \leq j \leq m, t \geq 0$, we have $\left|G^{i}\right|^{1 / 2}, F_{j}^{i} \in \mathrm{L}^{2}[0, T], H^{i} \in$ $\mathbb{H}_{\lambda}^{2}([0, T] \times Z ; E)$ and $K$ is predictable:

$$
\begin{aligned}
Y^{i}(t)= & Y^{i}(0)+\int_{0}^{t} G^{i}(s) d s+\int_{0}^{t} F_{j}^{i}(s) d B^{j}(s)+\int_{0}^{t} \int_{|z|<1} H^{i}(s, z) \tilde{N}(d s, d z) \\
& +\int_{0}^{t} \int_{|z| \geq 1} K^{i}(s, z) N(d s, d z)
\end{aligned}
$$

Here $B$ is an $m$-dimensional standard Brownian motion and $N$ is an independent Poisson random measure on $\mathbb{R}^{+} \times\left(\mathbb{R}^{d}-\{0\}\right)$ with compensator $\tilde{N}$ and intensity measure $\lambda$, which is a Lévy measure.

We often simplify complicated expressions by employing the notation of stochastic differentials to represent Lévy-type stochastic integrals. We then write $(2.3)$ as

$$
d Y(t)=G(t) d t+F(t) d B(t)+H(t, z) \tilde{N}(d t, d z)+K(t, z) N(d t, d z) .
$$

When we want particularly to emphasize the domain of integration with respect to $\mathrm{z}$, we will use the equivalent notation

$$
\begin{aligned}
d Y(t)= & G(t) d t+F(t) d B(t)+\int_{|z|<1} H(t, z) \tilde{N}(d t, d x) \\
& +\int_{|z| \geq 1} K(t, z) N(d t, d z) .
\end{aligned}
$$

Clearly $\mathrm{Y}$ is a semi martingale.

Let $\mathrm{Y}$ be a general Lévy-type stochastic process with stochastic differential

$$
\begin{aligned}
d Y^{i}(t)= & G^{i}(t) d t+F_{j}^{i}(t) d B^{j}(t)+\int_{|z|<1} H^{i}(t, z) \tilde{N}(d t, d z) \\
& +\int_{|z| \geq 1} K^{i}(t, z) N(d t, d z) .
\end{aligned}
$$

where, for each $1 \leq i \leq d, 1 \leq j \leq m, t \geq 0,\left|G^{i}\right|^{1 / 2}, F_{j}^{i} \in \mathrm{L}^{2}[0, T]$ and $H^{i} \in \mathbb{H}_{\lambda}^{2}([0, T] \times Z ; E)$. Let

$$
d Y_{c}(t)=G^{i}(t) d t+F_{j}^{i}(t) d B^{j}(t),
$$

and the discontinuous part of $Y$

$$
d Y_{d}(t)=\int_{|z|<1} H^{i}(t, z) \tilde{N}(d t, d z)+\int_{|z| \geq 1} K^{i}(t, z) N(d t, d z)
$$


so that for each $t \geq 0$

$$
Y(t)=Y(0)+Y_{c}(t)+Y_{d}(t) .
$$

Assumption 2.17. For all $t>0$,

$$
\sup _{0 \leq s \leq t} \sup _{0<|z|<1}|H(s, z)|<\infty \quad \text { a.s }
$$

Lemma 2.18. (Itô's theorem 1) If $Y=\left(Y_{t}\right)_{t>0}$ is a Lévy-type stochastic integral of the form (2.4), then, for each $f \in C^{2}\left(\mathbb{R}^{d}\right), t \geq 0$, with probability 1 we have

$$
\begin{aligned}
f(Y(t))-f(Y(0)) & \\
= & \int_{0}^{t} \partial_{i} f(Y(s-)) d Y_{c}^{i}(s)+\frac{1}{2} \int_{0}^{t} \partial_{i} \partial_{j} f(Y(s-)) d\left[Y_{c}^{i}, Y_{c}^{j}\right](s) \\
& +\int_{0}^{t} \int_{|z| \geq 1}[f(Y(s-)+K(s, z))-f(Y(s-))] N(d s, d z) \\
& +\int_{0}^{t} \int_{|z|<1}[f(Y(s-)+H(s, z))-f(Y(s-))] \tilde{N}(d s, d z) \\
& +\int_{0}^{t} \int_{|z|<1}[f(Y(s-)+H(s, z))-f(Y(s-)) \\
& \left.-H^{i}(s, z) \partial_{i} f(Y(s-))\right] \lambda(d z) d s .
\end{aligned}
$$

For proof see Theorem 4.4.7 of [5].

Definition 2.19. Let $M$ be a Brownian integral with the drift of the form

$$
M^{i}(t)=\int_{0}^{t} F_{j}^{i}(s) d B^{j}(s)+\int_{0}^{t} G^{i}(s) d s,
$$

where each $F_{j}^{i},\left(G^{i}\right)^{1 / 2} \in \mathrm{L}^{2}[0, T]$ for all $t \geq 0,1 \leq i \leq d, 1 \leq j \leq m$.

For each $1 \leq i \leq j$, the quadratic variation process, denoted as $\left(\left[M^{i}, M^{j}\right](t), t \geq 0\right)$, is defined by

$$
\left[M^{i}, M^{j}\right](t)=\sum_{k=1}^{m} \int_{0}^{t} F_{k}^{i}(s) F_{k}^{j}(s) d s .
$$

Lemma 2.20. (Burkholder's Inequality) Let $M=(M(t), t \geq 0)$ be a (real-valued) Brownian integral of the form

$$
M(t)=\int_{0}^{t} F^{j}(s) d B_{j}(s),
$$

where each $F^{j} \in \mathrm{L}^{2}[0, T], 1 \leq j \leq d, t \geq 0$. Let

$$
[M, M](t)=\sum_{j=1}^{m} \int_{0}^{t} F_{j}(s)^{2} d s,
$$

for each $t \geq 0$. Then $M$ is a square-integrable martingale. Let $\mathbb{E}\left([M, M](t)^{p / 2}\right)$ $<\infty$, then for any $p \geq 2$ there exists a $C(p)>0$ such that, for each $t \geq 0$, 


$$
\mathbb{E}\left(|M(t)|^{p}\right) \leq C(p) \mathbb{E}\left([M, M](t)^{p / 2}\right) .
$$

For proof see Theorem 4.4.21 of [5].

Lemma 2.21. (Burkholder-Davis-Gundy Inequality) For every $p \geq 1$, there is a constant $C_{p} \in(0, \infty)$ such that for any real-valued square integrable càdlàg martingale $M$ with $M_{0}=0$, and for any $T \geq 0$,

$$
C_{p}^{-1} \mathbb{E}[M, M]_{T}^{p / 2} \leq \mathbb{E} \sup _{0 \leq t \leq T}\left|M_{t}\right|^{p} \leq C_{p} \mathbb{E}[M, M]_{T}^{p / 2} .
$$

For proof see Theorem 3.50 of [26]

Definition 2.22. Let $g: \mathbb{R}^{+} \times Z \times \Omega \rightarrow F$ be given. A sequence $\left\{g_{n}\right\}_{n \in \mathbb{N}}$ of $F_{T} / \mathcal{B}(F)$ measurable functions is $\mathrm{L}^{p}$ - approximating $g$ on $(0, T] \times Z \times \Omega$ w.r.t. $\lambda \otimes \mathrm{P}$, if $g_{n}$ is $\lambda \otimes \mathrm{P}$-a.s. converging to $g$, when $n \rightarrow \infty$, and

$$
\lim _{n \rightarrow \infty} \int_{0}^{T} \int_{Z} \mathbb{E}\left[\left\|g_{n}(t, z, \omega)-g(t, z, \omega)\right\|^{p}\right] \mathrm{d} \lambda=0,
$$

i.e., $\left\|g_{n}-g\right\|$ converges to zero in $\mathrm{L}^{p}((0, T] \times Z \times \Omega, \lambda \otimes \mathrm{P})$, when $n \rightarrow \infty$.

Definition 2.23. Let $p \geq 1, T>0$. We say that $g$ is strong $p$-integrable on $(0, T] \times Z, Z \in \mathcal{B}(E)$, if there exists a sequence $\left\{g_{n}\right\}_{n \in N} \in \Sigma(E)$ of simple functions, such that $g_{n}$ is $\mathrm{L}^{p}$-approximating $g$ on $(0, T] \times Z \times \Omega$ w.r.t. $\lambda \otimes P$, and for any such sequence the limit of the natural integrals of $g_{n}$ w.r.t. $\tilde{N}(d t, d z)$ exists in $\mathrm{L}^{p}(\Omega, \mathcal{F}, P)$ for $n \rightarrow \infty$, i.e.,

$$
\int_{0}^{T} \int_{Z} g(t, z, \omega) \tilde{N}(d t, d z)(\omega)=\lim _{n \rightarrow \infty}^{p} \int_{0}^{T} \int_{Z} g_{n}(t, z, \omega) \tilde{N}(d t, d z)(\omega)
$$

exists. Moreover, the limit (2.6) does not depend on the sequence $\left\{g_{n}\right\}_{n \in \mathbb{N}} \in$ $\Sigma(E)$, which is $\mathrm{L}^{p}$-approximating $g$ on $(0, T] \times Z \times \Omega$ w.r.t. $\lambda \otimes P$. We call the limit in (2.6) the strong $p$-integral of $g$ w.r.t. $\tilde{N}(d t, d z)$ on $(0, T] \times Z$.

Lemma 2.24. Let $p \geq 1$. Let $g$ be strong $p$-integrable on $(0, T] \times Z, Z \in \mathcal{B}(E)$. Then the strong p-integral $\int_{0}^{t} \int_{Z} g(s, z, \omega) \tilde{N}(d s, d z)(\omega), t \in[0, T]$, is an $\mathcal{F}_{t^{-}}$ martingale with mean zero.

For proof see Theorem 4.10 of [28].

Lemma 2.25. Let $f \in \mathrm{L}^{1}([0, T], E)$; then $f$ is strong 1-integrable w.r.t $\tilde{N}(d t, d z)$ on $(0, T] \times Z$, for any $0<t \leq T, Z \in \mathcal{B}(E)$. Moreover

$$
\mathbb{E}\left[\left\|\int_{0}^{t} \int_{Z} f(s, z, \omega) \tilde{N}(d s, d z)\right\|\right] \leq 2 \int_{0}^{t} \int_{Z} \mathbb{E}[\|f(s, z, \omega)\|] \lambda(d z) d s
$$

For proof see Theorem 4.12 of [28].

Lemma 2.26. Suppose that $(F, \mathcal{B}(F))=(H, \mathcal{B}(H))$ is a separable Hilbert space. Let $g \in \mathbb{H}_{\lambda}^{2}(E)$, then $g$ is strong 2-integrable w.r.t. $\tilde{N}(d t, d z)$ on $(0, T] \times Z$, for any $0<t \leq T, Z \in \mathcal{B}(E)$. Moreover

$\mathbb{E}\left[\left\|\int_{0}^{t} \int_{Z} g(s, z, \omega) \tilde{N}(d s, d z)(\omega)\right\|^{2}\right]=\int_{0}^{t} \int_{Z} \mathbb{E}\left[\| g\left(s, z, \omega \|^{2}\right] \lambda(d s) d z\right.$ 
For proof see Theorem 4.14 of [28].

Lemma 2.27. Let $1<p \leq 2$ and let $E$ be a separable Banach space. Assume that $g \in \mathbb{H}_{\lambda}^{p}((0, \infty) \times Z ; E)$. Then there exists a constant $C=C_{p}(E) 2^{2-p}$ only depending on $E$ and $p$ such that for $0<q \leq p$

$\mathbb{E} \sup _{t \in[0, T]}\left|\int_{0}^{t} \int_{Z} g(s, z, \omega) \tilde{N}(d s, d z)\right|^{q} \leq C \mathbb{E}\left(\int_{0}^{T} \int_{Z}|g(t, z, \omega)|^{p} \lambda(d z) d t\right)^{q / p}$.

For proof see Corollary C.2 of [8] and Proposition 2.4 of [14].

\section{The stochastic GOY model of turbulence}

The GOY model (Gledger-Ohkitani-Yamada) [25] is a particular case of so called "Shell model" (see [12]). This model is the Navier-Stokes equation written in the Fourier space where the interaction between different modes is preserved between nearest modes. To be precise, the GOY model describes a one-dimensional cascade of energies among an infinite sequence of complex velocities, $\left\{u_{n}(t)\right\}$, on a one dimensional sequence of wave numbers

$$
k_{n}=k_{0} 2^{n}, \quad k_{0}>0, n=1,2, \ldots
$$

where the discrete index $n$ is referred to as the "shell index". The equations of motion of the GOY model of turbulence have the form

$$
\begin{aligned}
& \frac{\mathrm{d} u_{n}}{\mathrm{~d} t}+\nu k_{n}^{2} u_{n}+i\left(a k_{n} u_{n+1}^{\star} u_{n+2}^{\star}+b k_{n-1} u_{n-1}^{\star} u_{n+1}^{\star}\right. \\
& \left.\quad+c k_{n-2} u_{n-1}^{\star} u_{n-2}^{\star}\right)=f_{n}, \quad \text { for } n=1,2, \ldots,
\end{aligned}
$$

along with the boundary conditions

$$
u_{-1}=u_{0}=0 .
$$

Here $u_{n}^{\star}$ denotes the complex conjugate of $u_{n}, \nu>0$ is the kinematic viscosity and $f_{n}$ is the Fourier component of the forcing. $a, b$ and $c$ are real parameters such that energy conservation condition $a+b+c=0$ holds (see $[16,25])$.

\subsection{Functional setting}

Let $H$ be a real Hilbert space such that

$$
H:=\left\{u=\left(u_{1}, u_{2}, \ldots\right) \in \mathbb{C}^{\infty}: \sum_{n=1}^{\infty}\left|u_{n}\right|^{2}<\infty\right\} .
$$

For every $u, v \in H$, the scalar product $(\cdot, \cdot)$ and norm $|\cdot|$ are defined on $H$ as

$$
(u, v)_{H}=\operatorname{Re} \sum_{n=1}^{\infty} u_{n} v_{n}^{\star}, \quad|u|=\left(\sum_{n=1}^{\infty}\left|u_{n}\right|^{2}\right)^{1 / 2} .
$$

Let us now define the space

$$
V:=\left\{u \in H: \sum_{n=1}^{\infty} k_{n}^{2}\left|u_{n}\right|^{2}<\infty\right\}
$$


which is a Hilbert space equipped with the norm

$$
\|u\|=\left(\sum_{n=1}^{\infty} k_{n}^{2}\left|u_{n}\right|^{2}\right)^{1 / 2} .
$$

The linear operator $A: D(A) \rightarrow H$ is a positive definite, self adjoint linear operator defined by

$$
A u=\left((A u)_{1},(A u)_{2}, \ldots\right), \quad \text { where }(A u)_{n}=k_{n}^{2} u_{n}, \forall u \in D(A) .
$$

The domain of $A, D(A) \subset H$, is a Hilbert space equipped with the norm

$$
\|u\|_{D(A)}=|A u|=\left(\sum_{n=1}^{\infty} k_{n}^{4}\left|u_{n}\right|^{2}\right)^{1 / 2}, \quad \forall u \in D(A) .
$$

Since the operator $A$ is positive definite, we can define the power $A^{1 / 2}$,

$$
A^{1 / 2} u=\left(k_{1} u_{1}, k_{2} u_{2}, \ldots\right), \quad \forall u=\left(u_{1}, u_{2}, \ldots\right) .
$$

Furthermore, we define the space

$$
D\left(A^{1 / 2}\right)=\left\{u=\left(u_{1}, u_{2}, \ldots\right): \sum_{n=1}^{\infty} k_{n}^{2}\left|u_{n}\right|^{2}<\infty\right\}
$$

which is a Hilbert space equipped with the scalar product

$$
(u, v)_{D\left(A^{1 / 2}\right)}=\left(A^{1 / 2} u, A^{1 / 2} v\right), \quad \forall u, v \in D\left(A^{1 / 2}\right),
$$

and the norm

$$
\|u\|_{D\left(A^{1 / 2}\right)}=\left(\sum_{n=1}^{\infty} k_{n}^{2}\left|u_{n}\right|^{2}\right)^{1 / 2} .
$$

Note that $V=D\left(A^{1 / 2}\right)$. We consider $V^{\prime}=D\left(A^{-1 / 2}\right)$ as the dual space of $V$. Then the following inclusion holds

$$
V \subset H=H^{\prime} \subset V^{\prime} \text {. }
$$

We will now introduce the sequence spaces analogue to Sobolev functional spaces. For $1 \leq p<\infty$ and $s \in \mathbb{R}$

$$
\mathrm{W}^{s, p}:=\left\{u=\left(u_{1}, u_{2}, \ldots\right):\left\|A^{s / 2} u\right\|_{p}=\left(\sum_{n=1}^{\infty}\left(k_{n}^{s}\left|u_{n}\right|\right)^{p}\right)^{1 / p}<\infty\right\},
$$

and for $p=\infty$

$$
\mathrm{W}^{s, \infty}:=\left\{u=\left(u_{1}, u_{2}, \ldots\right):\left\|A^{s / 2} u\right\|_{\infty}=\sup _{1 \leq n<\infty}\left(k_{n}^{s}\left|u_{n}\right|\right)<\infty\right\},
$$

where for $u \in \mathrm{W}^{s, p}$ the norm is defined as

$$
\|u\|_{\mathrm{W}^{s, p}}=\left\|A^{s / 2} u\right\|_{p} .
$$

Here $\|\cdot\|$ denotes the usual norm in the $l^{p}$ sequence space. It is clear from the above definitions that $W^{1,2}=V=D\left(A^{1 / 2}\right)$. 
Remark 3.1. For the shell model we can reasonably assume that the complex velocities $u_{n}$ are such that $\left|u_{n}\right|<1$ for almost all $n$. Then

$$
\|u\|_{l^{4}}^{4}=\sum_{n=1}^{\infty}\left|u_{n}\right|^{4} \leq\left(\sum_{n=1}^{\infty}\left|u_{n}\right|^{2}\right)^{2}=|u|^{4}
$$

which leads to $H \subset l^{4}$.

We now state a Lemma which is useful in this work. We omit the proof since it is quite simple.

Lemma 3.2. For any smooth function $u \in H$, the following holds:

$$
\|u\|_{l^{4}}^{4} \leq C|u|^{2}\|u\|^{2} .
$$

\subsection{Properties of the linear and nonlinear operators}

We define the bilinear operator $B(\cdot, \cdot): V \times H \rightarrow H$ as

$$
B(u, v)=\left(B_{1}(u, v), B_{2}(u, v), \ldots\right),
$$

where

$$
B_{n}(u, v)=i k_{n}\left(\frac{1}{4} u_{n+1}^{\star} v_{n-1}^{\star}-\frac{1}{2}\left(u_{n+1}^{\star} v_{n+2}^{\star}+u_{n+2}^{\star} v_{n+1}^{\star}\right)+\frac{1}{8} u_{n-1}^{\star} v_{n-2}^{\star}\right) .
$$

In other words, if $\left\{e_{n}\right\}_{n=1}^{\infty}$ be a orthonormal basis of $H$, i.e. all the entries of $e_{n}$ are zero except at the place $n$ it is equal to 1 , then

$$
B(u, v)=i \sum_{n=1}^{\infty} k_{n}\left(\frac{1}{4} u_{n+1}^{\star} v_{n-1}^{\star}-\frac{1}{2}\left(u_{n+1}^{\star} v_{n+2}^{\star}+u_{n+2}^{\star} v_{n+1}^{\star}\right)+\frac{1}{8} u_{n-1}^{\star} v_{n-2}^{\star}\right) e_{n} .
$$

The following lemma says that $B(u, v)$ makes sense as an element of $H$, whenever $u \in V$ and $v \in H$ or $u \in H$ and $v \in V$. It also says that $B(u, v)$ makes sense as an element of $V^{\prime}$. Here we state the following lemma which has been proved in Constantin, Levant and Titi [9] for the Sabra shell model, but one can also prove the similar estimates for the GOY model (see [6]).

Lemma 3.3. (i) There exist constants $C_{1}>0, C_{2}>0$,

$$
|B(u, v)| \leq C_{1}\|u\||v|, \quad \forall u \in V, \quad v \in H,
$$

and

$$
|B(u, v)| \leq C_{2}|u|\|v\|, \quad \forall u \in H, \quad v \in V .
$$

(ii) $B: H \times H \rightarrow V^{\prime}$ is a bounded bilinear operator and for a constant $C_{3}>0$

$$
\|B(u, v)\|_{V^{\prime}} \leq C_{3}|u \| v|, \quad \forall u, \quad v \in H .
$$

(iii) $B: H \times D(A) \rightarrow V$ is a bounded bilinear operator and for a constant $C_{4}>0$

$$
\|B(u, v)\|_{V} \leq C_{4}|u \|| A v \mid, \quad \forall u \in H, \quad v \in D(A) .
$$

(iv) For every $u \in V$ and $v \in H$

$$
(B(u, v), v)=0 .
$$


We now present one more important property of the nonlinear operator $B$ in the following lemma which will play important role in the later part of this section. The proof is straightforward and uses the bilinearity property of $B$.

Lemma 3.4. If $w=u-v$, then

$$
B(u, u)-B(v, v)=B(v, w)+B(w, v)+B(w, w) .
$$

With above functional setting and following the classical treatment of the Navier-Stokes equation, one can write the stochastic GOY model of turbulence (3.1) with the Lévy forcing as the following,

$$
\mathrm{d} u+[\nu A u+B(u, u)] \mathrm{d} t=f(t) \mathrm{d} t+\sqrt{\varepsilon} \sigma(t, u) \mathrm{d} W(t)+\varepsilon \int_{Z} g(u, z) \tilde{N}(d t, d z)
$$

$$
u(0)=u_{0},
$$

where $u \in H$, the operators $A$ and $B$ are defined through (3.3) and (3.5) respectively, $f=\left(f_{1}, f_{2}, \ldots\right), \sigma(t, u)=\left(\sigma_{1}\left(t, u_{1}\right), \sigma_{2}\left(t, u_{2}\right), \ldots\right)$. Here $\left(W(t)_{t \geq 0}\right)$ is a $H$-valued Wiener process with trace class covariance, and the space $L_{Q}$ has been defined in Sect. 1. Here $g(u, z)$ is a measurable mapping from $H \times Z$ into $\mathrm{H}$ and let $\mathcal{D}([0, T], H)$ be the space of all càdlàg paths from $[0, T]$ into $\mathrm{H}$.

Assume that $\sigma$ and $g$ satisfy the following hypotheses of joint continuity, Lipschitz condition and linear growth:

Hypothesis 3.5. The main hypothesis is the following,

H.1. The function $\sigma \in C\left([0, T] \times V ; L_{Q}\left(H_{0} ; H\right)\right)$, and $g \in \mathbb{H}_{\lambda}^{2}([0, T] \times Z ; H)$.

H.2. For all $t \in(0, T)$, there exists a positive constant $K$ such that for all $u \in H$,

$$
|\sigma(t, u)|_{L_{Q}}^{2}+\int_{Z}|g(u, z)|_{H}^{2} \lambda(d z) \leq K\left(1+|u|^{2}\right) .
$$

H.3. For all $t \in(0, T)$, there exists a positive constant $L$ such that for all $u, v \in H$,

$$
|\sigma(t, u)-\sigma(t, v)|_{L_{Q}}^{2}+\int_{Z}|g(u, z)-g(v, z)|_{H}^{2} \lambda(d z) \leq L|u-v|^{2}
$$

The following lemma shows that sum of the linear and nonlinear operator is locally monotone in the $l^{4}$-ball.

Lemma 3.6. For a given $r>0$, let us denote by $\mathbb{B}_{r}$ the closed $l^{4}$-ball in $V$ :

$$
\mathbb{B}_{r}=\left\{v \in V ;\|v\|_{l^{4}} \leq r\right\} .
$$

Define the nonlinear operator $F$ on $V$ by $F(u):=-\nu A u-B(u, u)$. Then for $0<\varepsilon<\frac{\nu}{2 L}$, where $L$ is the positive constant that appears in the condition (H.3), the pair $\left(F, \sqrt{\varepsilon} \sigma+\varepsilon \int_{Z} g(., z) \lambda(d z)\right)$ is monotone in $\mathbb{B}_{r}$, i.e. for any $u \in V$ and $v \in \mathbb{B}_{r}$

$$
\begin{aligned}
& (F(u)-F(v), w)-\frac{r^{4}}{\nu^{3}}|w|^{2} \\
& \quad+\varepsilon\left[|\sigma(t, u)-\sigma(t, v)|_{L_{Q}}^{2}+\int_{Z}|g(u, z)-g(v, z)|^{2} \lambda(d z)\right] \leq 0
\end{aligned}
$$


where $w=u-v$.

Proof. First note that,

$$
\nu(A w, w)=\nu\|w\|^{2} .
$$

Next using the Lemma 3.4 and Eq. (3.10) from Lemma 3.3, we have

$$
(B(u, u)-B(v, v), w)=(B(v, w)+B(w, v)+B(w, w), w)=(B(w, v), w) .
$$

Now using the definition of the operator $B$ and Eq. (3.4) from Lemma 3.2, we get for $C>0$,

$$
\begin{aligned}
|(B(w, v), w)|= & \mid \sum_{n=1}^{\infty} i k_{n}\left[\frac{1}{4} v_{n-1}^{\star} w_{n+1}^{\star} w_{n}^{\star}-\frac{1}{2}\left(w_{n+1}^{\star} v_{n+2}^{\star}+w_{n+2}^{\star} v_{n+1}^{\star}\right) w_{n}^{\star}+\right. \\
& \left.+\frac{1}{8} w_{n-1}^{\star} v_{n-2}^{\star} w_{n}^{\star}\right] \mid \\
\leq & C\|v\|_{l^{4}}\|w\|_{l^{4}}\|w\| \\
\leq & \|v\|_{l^{4}}|w|^{1 / 2}\|w\|^{3 / 2} \\
\leq & \frac{\nu}{2}\|w\|^{2}+\frac{27}{32 \nu^{3}}|w|^{2}\|v\|_{l^{4}}^{4}
\end{aligned}
$$

Since $v \in \mathbb{B}_{r}$, the above relation yields

$$
-(B(w, v), w) \leq \frac{\nu}{2}\|w\|^{2}+\frac{r^{4}}{\nu^{3}}|w|^{2} .
$$

Hence by the definition of the operator $F$,

$$
(F(u)-F(v), w) \leq-\frac{\nu}{2}\|w\|^{2}+\frac{r^{4}}{\nu^{3}}|w|^{2} .
$$

We have

$$
(F(u)-F(v), w)+\frac{\nu}{2}\|w\|^{2}-\frac{r^{4}}{\nu^{3}}|w|^{2} \leq 0 .
$$

But $V \subset H \Rightarrow \frac{\nu}{2}|w|^{2} \leq \frac{\nu}{2}\|w\|^{2}$. We get,

$$
(F(u)-F(v), w)+\frac{\nu}{2}|w|^{2}-\frac{r^{4}}{\nu^{3}}|w|^{2} \leq 0 .
$$

Using condition (H.3) one can deduce that,

$$
\begin{aligned}
& (F(u)-F(v), w)-\frac{r^{4}}{\nu^{3}}|w|^{2}+\frac{\nu}{2 L}\left[|\sigma(t, u)-\sigma(t, v)|_{L_{Q}}^{2}\right. \\
& \left.\quad+\int_{Z}|g(u, z)-g(v, z)|^{2} \lambda(d z)\right] \leq 0
\end{aligned}
$$

Now choose $0<\varepsilon<\frac{\nu}{2 L}$ so that we get,

$$
\begin{aligned}
& (F(u)-F(v), w)-\frac{r^{4}}{\nu^{3}}|w|^{2} \\
& \quad+\varepsilon\left[|\sigma(t, u)-\sigma(t, v)|_{L_{Q}}^{2}+\int_{Z}|g(u, z)-g(v, z)|^{2} \lambda(d z)\right] \leq 0
\end{aligned}
$$




\section{Energy estimates and existence result}

Let $H_{n}:=\operatorname{span}\left\{e_{1}, e_{2}, \ldots, e_{n}\right\}$ where $\left\{e_{j}\right\}$ is any fixed orthonormal basis in $H$ with each $e_{j} \in D(A)$. Let $P_{n}$ denote the orthogonal projection of $H$ to $H_{n}$. Define $u^{n}=P_{n} u$, not to cause any confusion in notation with earlier $u_{n}$. Let $W_{n}=P_{n} W$. Let $\sigma_{n}=P_{n} \sigma$ and $\int_{Z} g^{n}\left(u^{n, \varepsilon}(t-), z\right) \tilde{N}(d t, d z)=$ $P_{n} \int_{Z} g(u(t-), z) \tilde{N}(d t, d z)$, where $g^{n}=P_{n} g$. Define $u^{n, \varepsilon}$ as the solution of the following stochastic differential equation in the variational form such that for each $v \in H_{n}$

$$
\begin{aligned}
\mathrm{d}\left(u^{n, \varepsilon}(t), v\right)= & \left(F\left(u^{n, \varepsilon}(t)\right), v\right) \mathrm{d} t+(f(t), v) \mathrm{d} t+\sqrt{\varepsilon}\left(\sigma_{n}\left(t, u^{n, \varepsilon}(t)\right) \mathrm{d} W_{n}(t), v\right) \\
& +\varepsilon \int_{Z}\left(g^{n}\left(u^{n, \varepsilon}(t-), z\right), v\right) \tilde{N}(d t, d z)
\end{aligned}
$$

with $u^{n, \varepsilon}(0)=P_{n} u(0)$.

Theorem 4.1. Under the above mathematical setting let $f$ be in $\mathrm{L}^{2}([0, T], H)$, $u(0)$ be $\mathcal{F}_{0}$ measurable, $\sigma \in C\left([0, T] \times V ; L_{Q}\left(H_{0} ; H\right)\right), g \in \mathbb{H}_{\lambda}^{2}([0, T] \times Z ; H)$ and $\mathbb{E}|u(0)|^{2}<\infty$. Let $u^{n, \varepsilon}$ denote the unique strong solution of the stochastic differential equation (4.1) in $\mathcal{D}\left([0, T], H_{n}\right)$. Then with $K$ as in condition (H.2), the following estimates hold:

For all $\varepsilon$, and $0 \leq t \leq T$,

$$
\begin{aligned}
& \mathbb{E}\left|u^{n, \varepsilon}(t)\right|^{2}+\nu \int_{0}^{t} \mathbb{E}\left\|u^{n, \varepsilon}(s)\right\|^{2} \mathrm{~d} s \\
& \leq\left(1+\varepsilon K T e^{\varepsilon K T}\right)\left(\mathbb{E}|u(0)|^{2}+\frac{1}{\nu} \int_{0}^{t}\|f(s)\|_{V^{\prime}}^{2} d s+\varepsilon K T\right),
\end{aligned}
$$

and for all $\varepsilon>0$,

$\mathbb{E}\left[\sup _{0 \leq t \leq T}\left|u^{n, \varepsilon}(t)\right|^{2}+2 \nu \int_{0}^{T}\left\|u^{n, \varepsilon}(t)\right\|^{2} \mathrm{~d} t\right] \leq C\left(\mathbb{E}|u(0)|^{2}, \int_{0}^{T}\|f(t)\|_{V^{\prime}}^{2} \mathrm{~d} t, \nu, T\right)$.

Proof. Applying Itô's lemma to the function $\left|u^{n, \varepsilon}(t)\right|^{2}$ and using the properties of the operators $A$ and $B$, we notice that,

$$
\begin{aligned}
\mathrm{d}\left|u^{n, \varepsilon}(t)\right|^{2}+2 \nu\left\|u^{n, \varepsilon}(t)\right\|^{2} \mathrm{~d} t \\
=2\left(f(t), u^{n, \varepsilon}(t)\right) \mathrm{d} t+\varepsilon \operatorname{Tr}\left(\sigma_{n}\left(t, u^{n, \varepsilon}(t)\right) Q \sigma_{n}\left(t, u^{n, \varepsilon}(t)\right)\right) \mathrm{d} t \\
\quad+2 \sqrt{\varepsilon}\left(\sigma_{n}\left(t, u^{n, \varepsilon}(t)\right), u^{n, \varepsilon}(t)\right) \mathrm{d} W_{n}(t)+\varepsilon \int_{Z}\left|g^{n}\left(u^{n, \varepsilon}(s-), z\right)\right|^{2} N(d s, d z) \\
\quad+2 \varepsilon \int_{Z}\left(u^{n, \varepsilon}(s-), g^{n}\left(u^{n, \varepsilon}(s-), z\right)\right) \tilde{N}(d s, d z)
\end{aligned}
$$

Using the inequality

$$
2 a b \leq \delta a^{2}+\frac{1}{\delta} b^{2}
$$


on $2\left(f(t), u^{n, \varepsilon}(t)\right)$, we obtain

$$
\begin{aligned}
\mathrm{d}\left|u^{n, \varepsilon}(t)\right|^{2}+2 \nu\left\|u^{n, \varepsilon}(t)\right\|^{2} \mathrm{~d} t \leq & \left(\nu\left\|u^{n, \varepsilon}(t)\right\|^{2}+\frac{1}{\nu}\|f(t)\|_{V^{\prime}}^{2}\right) \mathrm{d} t \\
& +\varepsilon\left|\sigma_{n}\left(t, u^{n, \varepsilon}(t)\right)\right|^{2} \mathrm{~d} t \\
& +\varepsilon \int_{Z}\left|g^{n}\left(u^{n, \varepsilon}(s-), z\right)\right|^{2} N(d s, d z) \\
& +2 \sqrt{\varepsilon}\left(\sigma_{n}\left(t, u^{n, \varepsilon}(t)\right), u^{n, \varepsilon}(t)\right) \mathrm{d} W_{n}(t) \\
& +2 \varepsilon \int_{Z}\left(u^{n, \varepsilon}(s-), g^{n}\left(u^{n, \varepsilon}(s-), z\right)\right) \tilde{N}(d s, d z) .
\end{aligned}
$$

Define

$$
\tau_{N}=\inf \left\{t:\left|u^{n, \varepsilon}(t)\right|^{2}+\int_{0}^{t}\left\|u^{n, \varepsilon}(s)\right\|^{2} \mathrm{~d} s>N\right\} .
$$

Then integrating one can deduce

$$
\begin{aligned}
& \left|u^{n, \varepsilon}\left(t \wedge \tau_{N}\right)\right|^{2}+\nu \int_{0}^{t \wedge \tau_{N}}\left\|u^{n, \varepsilon}(s)\right\|^{2} \mathrm{~d} s \\
& \leq|u(0)|^{2}+\frac{1}{\nu} \int_{0}^{t \wedge \tau_{N}}\|f(s)\|_{V^{\prime}}^{2} \mathrm{~d} s+\int_{0}^{t \wedge \tau_{N}} \varepsilon\left|\sigma_{n}\left(s, u^{n, \varepsilon}(s)\right)\right|^{2} \mathrm{~d} s \\
& \quad+\int_{0}^{t \wedge \tau_{N}} \varepsilon \int_{Z}\left|g^{n}\left(u^{n, \varepsilon}(s-), z\right)\right|^{2} N(d s, d z) \\
& \quad+2 \sqrt{\varepsilon} \int_{0}^{t \wedge \tau_{N}}\left(\sigma_{n}\left(s, u^{n, \varepsilon}(s)\right), u^{n, \varepsilon}(s)\right) \mathrm{d} W_{n}(s) \\
& \quad+2 \int_{0}^{t \wedge \tau_{N}} \varepsilon \int_{Z}\left(u^{n, \varepsilon}(s-), g^{n}\left(u^{n, \varepsilon}(s-), z\right)\right) \tilde{N}(d s, d z)
\end{aligned}
$$

Hence we can write this as

$$
\begin{aligned}
& \left|u^{n, \varepsilon}\left(t \wedge \tau_{N}\right)\right|^{2}+\nu \int_{0}^{t \wedge \tau_{N}}\left\|u^{n, \varepsilon}(s)\right\|^{2} \mathrm{~d} s \\
& \leq|u(0)|^{2}+\frac{1}{\nu} \int_{0}^{t \wedge \tau_{N}}\|f(s)\|_{V^{\prime}}^{2} \mathrm{~d} s+\int_{0}^{t \wedge \tau_{N}} \varepsilon\left|\sigma_{n}\left(s, u^{n, \varepsilon}(s)\right)\right|^{2} \mathrm{~d} s \\
& \quad+\int_{0}^{t \wedge \tau_{N}} \varepsilon \int_{Z}\left|g^{n}\left(u^{n, \varepsilon}(s-), z\right)\right|^{2} \lambda(d z) d s \\
& \quad+2 \sqrt{\varepsilon} \int_{0}^{t \wedge \tau_{N}}\left(\sigma_{n}\left(s, u^{n, \varepsilon}(s)\right), u^{n, \varepsilon}(s)\right) \mathrm{d} W_{n}(s) \\
& \quad+\int_{0}^{t \wedge \tau_{N}} \varepsilon \int_{Z}\left|g^{n}\left(u^{n, \varepsilon}(s-), z\right)\right|^{2} \tilde{N}(d s, d z) \\
& \quad+2 \int_{0}^{t \wedge \tau_{N}} \varepsilon \int_{Z}\left(u^{n, \varepsilon}(s-), g^{n}\left(u^{n, \varepsilon}(s-), z\right)\right) \tilde{N}(d s, d z)
\end{aligned}
$$

Using Hölder's inequality, one can note that if $g^{n}$ is strong 2-integrable w.r.t $\tilde{N}(d t, d z),\left|g^{n}\right|^{2}$ is strong 1-integrable w.r.t $\tilde{N}(d t, d z)$. Hence taking expectation on both sides of (4.5), using the Lemma 2.25 on $\left|g^{n}\right|^{2}$, and using the fact 
that the stochastic integrals appeared in

$$
2 \sqrt{\varepsilon} \int_{0}^{t \wedge \tau_{N}}\left(\sigma_{n}\left(s, u^{n, \varepsilon}(s)\right), u^{n, \varepsilon}(s)\right) \mathrm{d} W_{n}(s)
$$

and

$$
2 \int_{0}^{t \wedge \tau_{N}} \varepsilon \int_{Z}\left(u^{n, \varepsilon}(s-), g^{n}\left(u^{n, \varepsilon}(s-), z\right)\right) \tilde{N}(d s, d z)
$$

are martingales, and having zero averages, we get

$$
\begin{aligned}
\mathbb{E} & {\left[\left|u^{n, \varepsilon}\left(t \wedge \tau_{N}\right)\right|^{2}+\nu \int_{0}^{t \wedge \tau_{N}}\left\|u^{n, \varepsilon}(s)\right\|^{2} \mathrm{~d} s\right] } \\
\leq & \mathbb{E}|u(0)|^{2}+\frac{1}{\nu} \int_{0}^{t \wedge \tau_{N}}\|f(s)\|_{V^{\prime}}^{2} \mathrm{~d} s \\
& +\int_{0}^{t \wedge \tau_{N}} \mathbb{E}\left[\varepsilon\left|\sigma_{n}\left(s, u^{n, \varepsilon}(s)\right)\right|^{2}\right] \mathrm{d} s \\
& +\int_{0}^{t \wedge \tau_{N}} \mathbb{E}\left[\varepsilon \int_{Z}\left|g^{n}\left(u^{n, \varepsilon}(s), z\right)\right|^{2} \lambda(d z)\right] \mathrm{d} s .
\end{aligned}
$$

Then we use the hypothesis (H.2) to obtain

$$
\begin{aligned}
\mathbb{E} & {\left[\left|u^{n, \varepsilon}\left(t \wedge \tau_{N}\right)\right|^{2}+\nu \int_{0}^{t \wedge \tau_{N}}\left\|u^{n, \varepsilon}(s)\right\|^{2} \mathrm{~d} s\right] } \\
& \leq \mathbb{E}|u(0)|^{2}+\frac{1}{\nu} \int_{0}^{t \wedge \tau_{N}}\|f(s)\|_{V^{\prime}}^{2} \mathrm{~d} s+\varepsilon K \int_{0}^{t \wedge \tau_{N}} \mathbb{E}\left(1+\left|u^{n, \varepsilon}(s)\right|^{2}\right) \mathrm{d} s .
\end{aligned}
$$

So finally we obtain

$$
\begin{aligned}
& \mathbb{E}\left[\left|u^{n, \varepsilon}\left(t \wedge \tau_{N}\right)\right|^{2}+\nu \int_{0}^{t \wedge \tau_{N}}\left\|u^{n, \varepsilon}(s)\right\|^{2} \mathrm{~d} s\right] \\
& \leq \mathbb{E}|u(0)|^{2}+\frac{1}{\nu} \int_{0}^{t \wedge \tau_{N}}\|f(s)\|_{V^{\prime}}^{2} \mathrm{~d} s+\varepsilon K T+\varepsilon K \int_{0}^{t \wedge \tau_{N}} \mathbb{E}\left(\left|u^{n, \varepsilon}(s)\right|^{2}\right) \mathrm{d} s .
\end{aligned}
$$

In particular

$$
\begin{aligned}
& \mathbb{E}\left[\left|u^{n, \varepsilon}\left(t \wedge \tau_{N}\right)\right|^{2}\right] \\
& \quad \leq \mathbb{E}|u(0)|^{2}+\frac{1}{\nu} \int_{0}^{t \wedge \tau_{N}}\|f(s)\|_{V^{\prime}}^{2} \mathrm{~d} s+\varepsilon K T+\varepsilon K \int_{0}^{t \wedge \tau_{N}} \mathbb{E}\left(\left|u^{n, \varepsilon}(s)\right|^{2}\right) \mathrm{d} s .
\end{aligned}
$$

Applying Gronwall's Inequality, we obtain

$$
\mathbb{E}\left[\left|u^{n, \varepsilon}\left(t \wedge \tau_{N}\right)\right|^{2}\right] \leq e^{\varepsilon K T}\left[\mathbb{E}|u(0)|^{2}+\frac{1}{\nu} \int_{0}^{t \wedge \tau_{N}}\|f(s)\|_{V^{\prime}}^{2} \mathrm{~d} s+\varepsilon K T\right] .
$$

So we get

$$
\begin{aligned}
& \mathbb{E}\left[\left|u^{n, \varepsilon}\left(t \wedge \tau_{N}\right)\right|^{2}\right]+\nu \int_{0}^{t \wedge \tau_{N}} \mathbb{E}\left\|u^{n, \varepsilon}(s)\right\|^{2} \mathrm{~d} s \\
& \leq\left(1+\varepsilon K T e^{\varepsilon K T}\right)\left(\mathbb{E}|u(0)|^{2}+\frac{1}{\nu} \int_{0}^{t \wedge \tau_{N}}\|f(s)\|_{V^{\prime}}^{2} d s+\varepsilon K T\right) .
\end{aligned}
$$

Taking the limit as $N \rightarrow \infty$ we have the result (4.2). 
To prove (4.3), we proceed in the similar way as above, but we take supremum upto time $T \wedge \tau_{N}$ before taking the expectation in equation (4.4),

$$
\begin{aligned}
\mathbb{E}\left[\sup _{0 \leq t \leq T \wedge \tau_{N}}\left|u^{n, \varepsilon}(t)\right|^{2}+\nu \int_{0}^{T \wedge \tau_{N}}\left\|u^{n, \varepsilon}(t)\right\|^{2} \mathrm{~d} t\right] \\
\leq \mathbb{E}|u(0)|^{2}+\frac{1}{\nu} \int_{0}^{T \wedge \tau_{N}}\|f(t)\|_{V^{\prime}}^{2} \mathrm{~d} t+\varepsilon K T+\varepsilon K \mathbb{E} \int_{0}^{T \wedge \tau_{N}} \sup _{0 \leq s \leq t}\left|u^{n, \varepsilon}(s)\right|^{2} \mathrm{~d} t \\
\quad+2 \sqrt{\varepsilon} \mathbb{E}\left[\sup _{0 \leq t \leq T \wedge \tau_{N}}\left|\int_{0}^{t}\left(\sigma_{n}\left(s, u^{n, \varepsilon}(s)\right), u^{n, \varepsilon}(s)\right) \mathrm{d} W_{n}(s)\right|\right] \\
\quad+2 \varepsilon \mathbb{E}\left[\sup _{0 \leq t \leq T \wedge \tau_{N}}\left|\int_{0}^{t} \int_{Z}\left(u^{n, \varepsilon}(s-), g^{n}\left(u^{n, \varepsilon}(s-), z\right)\right) \tilde{N}(d s, d z)\right|\right]
\end{aligned}
$$

Applying Burkholder-Davis-Gundy inequality, condition (H.2) and Young's inequality to the term

$$
2 \sqrt{\varepsilon} \mathbb{E}\left[\sup _{0 \leq t \leq T \wedge \tau_{N}}\left|\int_{0}^{t}\left(\sigma_{n}\left(s, u^{n, \varepsilon}(s)\right), u^{n, \varepsilon}(s)\right) \mathrm{d} W_{n}(s)\right|\right]
$$

we get,

$$
\begin{aligned}
& 2 \sqrt{\varepsilon} \mathbb{E}\left[\sup _{0 \leq t \leq T \wedge \tau_{N}}\left|\int_{0}^{t}\left(\sigma_{n}\left(s, u^{n, \varepsilon}(s)\right), u^{n, \varepsilon}(s)\right) \mathrm{d} W_{n}(s)\right|\right] \\
& \leq 2 \sqrt{2 \varepsilon} \mathbb{E}\left[\int_{0}^{T \wedge \tau_{N}}\left|\sigma\left(s, u^{n, \varepsilon}(s)\right)\right|^{2}\left|u^{n, \varepsilon}(s)\right|^{2} \mathrm{~d} s\right]^{1 / 2} \\
& \leq 2 \sqrt{2 \varepsilon K} \mathbb{E}\left[\left(\int_{0}^{T \wedge \tau_{N}}\left(1+\left|u^{n, \varepsilon}(t)\right|^{2}\right)\left|u^{n, \varepsilon}(t)\right|^{2} \mathrm{~d} t\right)^{1 / 2}\right] \\
& \leq 2 \sqrt{2 \varepsilon K} \mathbb{E}\left[\sup _{0 \leq t \leq T \wedge \tau_{N}}\left|u^{n, \varepsilon}(t)\right|\left(\int_{0}^{T \wedge \tau_{N}}\left(1+\left|u^{n, \varepsilon}(t)\right|^{2}\right) \mathrm{d} t\right)^{1 / 2}\right] \\
& {\left[\text { Young's inequality } a b \leq \eta a^{2}+C(\eta) b^{2} \quad(\eta>0)\right.} \\
& \text { for } \left.C(\eta)=\frac{1}{4 \eta} \text {, by taking } \eta=\frac{1}{8 \sqrt{2 \varepsilon K}}, C(\eta)=2 \sqrt{2 \varepsilon K}\right] \\
& \leq \frac{1}{4} \mathbb{E}\left(\sup _{0 \leq t \leq T \wedge \tau_{N}}\left|u^{n, \varepsilon}(t)\right|^{2}\right)+8 \varepsilon K \mathbb{E} \int_{0}^{T \wedge \tau_{N}}\left|u^{n, \varepsilon}(t)\right|^{2} \mathrm{~d} t+8 \varepsilon K T \\
& \leq \frac{1}{4} \mathbb{E}\left(\sup _{0 \leq t \leq T \wedge \tau_{N}}\left|u^{n, \varepsilon}(t)\right|^{2}\right)+8 \varepsilon K \mathbb{E} \int_{0}^{T \wedge \tau_{N}} \sup _{0 \leq s \leq t}\left|u^{n, \varepsilon}(s)\right|^{2} \mathrm{~d} t+8 \varepsilon K T .
\end{aligned}
$$


Now again by applying Burkholder-Davis-Gundy inequality in the form given in Lemma 2.27, condition (H.2) and Young's inequality to the term

$$
2 \varepsilon \mathbb{E}\left[\sup _{0 \leq t \leq T \wedge \tau_{N}}\left|\int_{0}^{t} \int_{Z}\left(u^{n, \varepsilon}(s-), g^{n}\left(u^{n, \varepsilon}(s-), z\right)\right) \tilde{N}(d s, d z)\right|\right]
$$

we get,

$$
\begin{aligned}
& 2 \varepsilon \mathbb{E}\left[\sup _{0 \leq t \leq T \wedge \tau_{N}}\left|\int_{0}^{t} \int_{Z}\left(u^{n, \varepsilon}(s-), g^{n}\left(u^{n, \varepsilon}(s-), z\right)\right) \tilde{N}(d s, d z)\right|\right] \\
& \leq 2 \sqrt{2} \varepsilon \mathbb{E}\left[\int_{0}^{T \wedge \tau_{N}} \int_{Z}\left|\left(u^{n, \varepsilon}(s), g^{n}\left(u^{n, \varepsilon}(s), z\right)\right)\right|^{2} \lambda(d z) d s\right]^{1 / 2} \\
& \leq 2 \sqrt{2} \varepsilon \mathbb{E}\left[\int_{0}^{T \wedge \tau_{N}} \int_{Z}\left|u^{n, \varepsilon}(s)\right|^{2}\left|g^{n}\left(u^{n, \varepsilon}(s), z\right)\right|^{2} \lambda(d z) d s\right]^{1 / 2} \\
& \leq 2 \sqrt{2} \varepsilon \mathbb{E}\left[\sup _{0 \leq t \leq T \wedge \tau_{N}}\left|u^{n, \varepsilon}(t)\right|\left(\int_{0}^{T \wedge \tau_{N}} \int_{Z}\left|g^{n}\left(u^{n, \varepsilon}(s), z\right)\right|^{2} \lambda(d z) d s\right)^{1 / 2}\right] \\
& \leq 2 \sqrt{2} \varepsilon \mathbb{E}\left[\sup _{0 \leq t \leq T \wedge \tau_{N}}\left|u^{n, \varepsilon}(t)\right|\left(\int_{0}^{T \wedge \tau_{N}} K\left(1+\left|u^{n, \varepsilon}(s)\right|^{2}\right) \mathrm{d} s\right)^{1 / 2}\right] \\
& {\left[\text { Young's inequality } a b \leq \eta a^{2}+C(\eta) b^{2} \quad(\eta>0)\right.} \\
& \text { for } \left.C(\eta)=\frac{1}{4 \eta} \text {, by taking } \eta=\frac{1}{8 \sqrt{2} \varepsilon}, C(\eta)=2 \sqrt{2} \varepsilon\right] \\
& 2 \varepsilon \mathbb{E}\left[\sup _{0 \leq t \leq T \wedge \tau_{N}}\left|\int_{0}^{t} \int_{Z}\left(u^{n, \varepsilon}(s-), g^{n}\left(u^{n, \varepsilon}(s-), z\right)\right) \tilde{N}(d s, d z)\right|\right] \\
& \leq \frac{1}{4} \mathbb{E}\left[\sup _{0 \leq t \leq T \wedge \tau_{N}}\left|u^{n, \varepsilon}(t)\right|^{2}\right]+8 \varepsilon^{2} K \mathbb{E} \int_{0}^{T \wedge \tau_{N}}\left|u^{n, \varepsilon}(t)\right|^{2} \mathrm{~d} t+8 \varepsilon^{2} K T \\
& \leq \frac{1}{4} \mathbb{E}\left[\sup _{0 \leq t \leq T \wedge \tau_{N}}\left|u^{n, \varepsilon}(t)\right|^{2}\right]+8 \varepsilon^{2} K \mathbb{E} \int_{0}^{T \wedge \tau_{N}} \sup _{0 \leq s \leq t}\left|u^{n, \varepsilon}(s)\right|^{2} \mathrm{~d} t+8 \varepsilon^{2} K T \text {. }
\end{aligned}
$$

Replace (4.7) and (4.8) in (4.6),

$$
\begin{aligned}
\mathbb{E}\left[\sup _{0 \leq t \leq T \wedge \tau_{N}}\left|u^{n, \varepsilon}(t)\right|^{2}\right]+2 \nu \int_{0}^{T \wedge \tau_{N}} \mathbb{E}\left\|u^{n, \varepsilon}(t)\right\|^{2} \mathrm{~d} t \\
\leq 2 \mathbb{E}|u(0)|^{2}+\frac{2}{\nu} \int_{0}^{T \wedge \tau_{N}}\|f(t)\|_{V^{\prime}}^{2} \mathrm{~d} t+2 \varepsilon K T(9+8 \varepsilon) \\
\quad+2 \varepsilon K(9+8 \varepsilon) \mathbb{E} \int_{0}^{T \wedge \tau_{N}} \sup _{0 \leq s \leq t}\left|u^{n, \varepsilon}(s)\right|^{2} \mathrm{~d} t
\end{aligned}
$$


Note $T \wedge \tau_{N} \rightarrow T$ a.s. as $N \rightarrow \infty$. Thus taking the limit in the above estimate (4.9) as $N \rightarrow \infty$, one can get for all $\varepsilon$

$$
\begin{aligned}
\mathbb{E}\left[\sup _{0 \leq t \leq T}\left|u^{n, \varepsilon}(t)\right|^{2}\right]+2 \nu \int_{0}^{T} \mathbb{E}\left\|u^{n, \varepsilon}(t)\right\|^{2} \mathrm{~d} t \\
\leq 2 \mathbb{E}|u(0)|^{2}+\frac{2}{\nu} \int_{0}^{T}\|f(t)\|_{V^{\prime}}^{2} \mathrm{~d} t+2 \varepsilon K T(9+8 \varepsilon) \\
\quad+2 \varepsilon K(9+8 \varepsilon) \mathbb{E} \int_{0}^{T} \sup _{0 \leq s \leq t}\left|u^{n, \varepsilon}(s)\right|^{2} \mathrm{~d} t .
\end{aligned}
$$

In particular

$$
\begin{aligned}
\mathbb{E}\left[\sup _{0 \leq t \leq T}\left|u^{n, \varepsilon}(t)\right|^{2}\right] \leq & 2 \mathbb{E}|u(0)|^{2}+\frac{2}{\nu} \int_{0}^{T}\|f(t)\|_{V^{\prime}}^{2} \mathrm{~d} t+2 \varepsilon K T(9+8 \varepsilon) \\
& +2 \varepsilon K(9+8 \varepsilon) \mathbb{E} \int_{0}^{T} \sup _{0 \leq s \leq t}\left|u^{n, \varepsilon}(s)\right|^{2} \mathrm{~d} t
\end{aligned}
$$

Now by applying Gronwall's Inequality, we obtain

$$
\begin{aligned}
& \mathbb{E}\left[\sup _{0 \leq t \leq T}\left|u^{n, \varepsilon}(t)\right|^{2}\right] \\
& \leq e^{2 \varepsilon K T(9+8 \varepsilon)}\left[2 \mathbb{E}|u(0)|^{2}+\frac{2}{\nu} \int_{0}^{T}\|f(t)\|_{V^{\prime}}^{2} \mathrm{~d} t+2 \varepsilon K T(9+8 \varepsilon)\right] .
\end{aligned}
$$

Now using by (4.12) in (4.10) one can deduce that

$$
\begin{aligned}
& \mathbb{E}\left[\sup _{0 \leq t \leq T}\left|u^{n, \varepsilon}(t)\right|^{2}\right]+2 \nu \int_{0}^{T} \mathbb{E}\left\|u^{n, \varepsilon}(t)\right\|^{2} \mathrm{~d} t \\
& \leq\left(1+2 \varepsilon K T(9+8 \varepsilon) e^{2 \varepsilon K T(9+8 \varepsilon)}\right) \\
& \times\left(2 \mathbb{E}|u(0)|^{2}+\frac{2}{\nu} \int_{0}^{T}\|f(t)\|_{V^{\prime}}^{2} \mathrm{~d} t+2 \varepsilon K T(9+8 \varepsilon)\right) .
\end{aligned}
$$

Hence, we obtain

$$
\begin{gathered}
\mathbb{E}\left[\sup _{0 \leq t \leq T}\left|u^{n, \varepsilon}(t)\right|^{2}\right]+2 \nu \int_{0}^{T} \mathbb{E}\left\|u^{n, \varepsilon}(t)\right\|^{2} \mathrm{~d} t \\
\leq C\left(\mathbb{E}|u(0)|^{2}, \int_{0}^{T}\|f(t)\|_{V^{\prime}}^{2} \mathrm{~d} t, \nu, T\right) .
\end{gathered}
$$

Theorem 4.2. Let $f$ be in $\mathrm{L}^{2}([0, T], H), u(0)$ be $\mathcal{F}_{0}$ measurable, $\sigma \in C([0, T] \times$ $\left.V ; L_{Q}\left(H_{0} ; H\right)\right), g \in \mathbb{H}_{\lambda}^{2}([0, T] \times Z ; H)$ and $\mathbb{E}|u(0)|^{2}<\infty$. Let $u^{n, \varepsilon}$ denote the unique strong solution of the stochastic differential equation (4.1) in $\mathcal{D}\left([0, T], H_{n}\right)$. Then with $K$ as in condition (H.2), the following estimates hold: 
For any $\delta>0$,

$$
\begin{aligned}
& \mathbb{E}\left|u^{n, \varepsilon}(t)\right|^{2} e^{-\delta t}+2 \nu \int_{0}^{T} \mathbb{E}\left\|u^{n, \varepsilon}(t)\right\|^{2} e^{-\delta t} \mathrm{~d} t \\
& \leq\left(1+\varepsilon K T e^{\varepsilon K T}\right)\left(\mathbb{E}|u(0)|^{2}+\frac{1}{\delta} \int_{0}^{T}|f(t)|^{2} e^{-\delta t} d t+\frac{\varepsilon K}{\delta}\right),
\end{aligned}
$$

and for any $\delta>0$,

$$
\begin{aligned}
& \mathbb{E}\left[\sup _{0 \leq t \leq T}\left|u^{n, \varepsilon}(t)\right|^{2} e^{-\delta t}\right]+4 \nu \int_{0}^{T} \mathbb{E}\left\|u^{n, \varepsilon}(t)\right\|^{2} e^{-\delta t} d t \\
& \leq C\left(\mathbb{E}|u(0)|^{2}, \int_{0}^{T}|f(t)|^{2} e^{-\delta t} d t, \delta, T\right) .
\end{aligned}
$$

Proof. In order prove this theorem we use the same method as in the previous theorem and also use the same stopping time argument.

We consider the function $e^{-\delta t}\left|u^{n, \varepsilon}(t)\right|^{2}$ for $\delta>0$ and apply the Itô Lemma to get,

$$
\begin{aligned}
\mathrm{d}[\mid & \left.\left.u^{n, \varepsilon}(t)\right|^{2} e^{-\delta t}\right]+2 \nu\left\|u^{n, \varepsilon}(t)\right\|^{2} e^{-\delta t} \mathrm{~d} t+\delta\left|u^{n, \varepsilon}(t)\right|^{2} e^{-\delta t} \mathrm{~d} t \\
= & {\left[2\left(f(t), u^{n, \varepsilon}(t)\right)+\varepsilon \operatorname{Tr}\left(\sigma_{n}\left(t, u^{n, \varepsilon}(t)\right) Q \sigma_{n}\left(t, u^{n, \varepsilon}(t)\right)\right)\right] e^{-\delta t} \mathrm{~d} t } \\
& +2 \sqrt{\varepsilon}\left(\sigma_{n}\left(t, u^{n, \varepsilon}(t)\right), u^{n, \varepsilon}(t)\right) e^{-\delta t} \mathrm{~d} W_{n}(t) \\
& +e^{-\delta t} \varepsilon \int_{Z}\left|g^{n}\left(u^{n, \varepsilon}(t-), z\right)\right|^{2} N(d t, d z) \\
& +2 e^{-\delta t} \int_{Z} \varepsilon\left(u^{n, \varepsilon}(t-), g^{n}\left(u^{n, \varepsilon}(t-), z\right)\right) \tilde{N}(d t, d z) .
\end{aligned}
$$

Note that

$$
2\left(f(t), u^{n, \varepsilon}(t)\right) \leq \delta\left|u^{n, \varepsilon}(t)\right|^{2}+\frac{1}{\delta}|f(t)|^{2} .
$$

So from the above relation we get

$$
\begin{aligned}
& \mathrm{d}\left[\left|u^{n, \varepsilon}(t)\right|^{2} e^{-\delta t}\right]+2 \nu\left\|u^{n, \varepsilon}(t)\right\|^{2} e^{-\delta t} \mathrm{~d} t \\
& \leq \frac{1}{\delta}|f(t)|^{2} e^{-\delta t} \mathrm{~d} t+\varepsilon \operatorname{Tr}\left(\sigma_{n}\left(t, u^{n, \varepsilon}(t)\right) Q \sigma_{n}\left(t, u^{n, \varepsilon}(t)\right)\right) e^{-\delta t} \mathrm{~d} t \\
&+2 \sqrt{\varepsilon}\left(\sigma_{n}\left(t, u^{n, \varepsilon}(t)\right), u^{n, \varepsilon}(t)\right) e^{-\delta t} \mathrm{~d} W_{n}(t) \\
&+e^{-\delta t} \int_{Z} \varepsilon\left|g^{n}\left(u^{n, \varepsilon}(t-), z\right)\right|^{2} N(d t, d z) \\
&+2 e^{-\delta t} \int_{Z} \varepsilon\left(u^{n, \varepsilon}(t-), g^{n}\left(u^{n, \varepsilon}(t-), z\right)\right) \tilde{N}(d t, d z) .
\end{aligned}
$$

Hence upon writing (4.16) in the integral form, then taking expectation and proceeding as in the previous stopping time given in the proof of Theorem 4.1 
one can get

$$
\begin{aligned}
& \mathbb{E}\left|u^{n, \varepsilon}(t)\right|^{2} e^{-\delta t}+2 \nu \mathbb{E} \int_{0}^{T}\left\|u^{n, \varepsilon}(t)\right\|^{2} e^{-\delta t} \mathrm{~d} t \\
& \leq \mathbb{E}|u(0)|^{2}+\frac{1}{\delta} \int_{0}^{T}|f(t)|^{2} e^{-\delta t} \mathrm{~d} t+\mathbb{E} \int_{0}^{T} \varepsilon\left|\sigma_{n}\left(t, u^{n, \varepsilon}(t)\right)\right|^{2} e^{-\delta t} \mathrm{~d} t \\
& \quad+\mathbb{E} \int_{0}^{T} e^{-\delta t} \int_{Z} \varepsilon\left|g^{n}\left(u^{n, \varepsilon}(t), z\right)\right|^{2} \lambda(d z) \mathrm{d} t
\end{aligned}
$$

Since the terms

$$
2 \sqrt{\varepsilon} \int_{0}^{T}\left(\sigma_{n}\left(t, u^{n, \varepsilon}(t)\right), u^{n, \varepsilon}(t)\right) e^{-\delta t} \mathrm{~d} W_{n}(t)
$$

and

$$
2 \int_{0}^{T} e^{-\delta t} \varepsilon \int_{Z}\left(u^{n, \varepsilon}(t-), g^{n}\left(u^{n, \varepsilon}(t-), z\right)\right) \tilde{N}(d t, d z)
$$

are martingales and having zero averages. Now applying (H.2) one can obtain

$$
\begin{aligned}
& \mathbb{E}\left|u^{n, \varepsilon}(t)\right|^{2} e^{-\delta t}+2 \nu \mathbb{E} \int_{0}^{T}\left\|u^{n, \varepsilon}(t)\right\|^{2} e^{-\delta t} \mathrm{~d} t \\
& \leq \mathbb{E}|u(0)|^{2}+\frac{1}{\delta} \int_{0}^{T}|f(t)|^{2} e^{-\delta t} \mathrm{~d} t+\frac{\varepsilon K}{\delta}+\varepsilon K \int_{0}^{T} \mathbb{E}\left|u^{n, \varepsilon}(t)\right|^{2} e^{-\delta t} \mathrm{~d} t .
\end{aligned}
$$

In particular

$$
\begin{aligned}
& \mathbb{E}\left|u^{n, \varepsilon}(t)\right|^{2} e^{-\delta t} \\
& \quad \leq \mathbb{E}|u(0)|^{2}+\frac{1}{\delta} \int_{0}^{T}|f(t)|^{2} e^{-\delta t} \mathrm{~d} t+\frac{\varepsilon K}{\delta}+\varepsilon K \int_{0}^{T} \mathbb{E}\left|u^{n, \varepsilon}(t)\right|^{2} e^{-\delta t} \mathrm{~d} t .
\end{aligned}
$$

Applying Gronwall's Inequality we get,

$$
\mathbb{E}\left|u^{n, \varepsilon}(t)\right|^{2} e^{-\delta t} \leq e^{\varepsilon K T}\left[\mathbb{E}|u(0)|^{2}+\frac{1}{\delta} \int_{0}^{T}|f(t)|^{2} e^{-\delta t} \mathrm{~d} t+\frac{\varepsilon K}{\delta}\right] .
$$

By using above relation in (4.18) one can deduce that

$$
\begin{aligned}
& \mathbb{E}\left|u^{n, \varepsilon}(t)\right|^{2} e^{-\delta t}+2 \nu \int_{0}^{T} \mathbb{E}\left\|u^{n, \varepsilon}(t)\right\|^{2} e^{-\delta t} \mathrm{~d} t \\
& \leq\left(1+\varepsilon K T e^{\varepsilon K T}\right)\left(\mathbb{E}|u(0)|^{2}+\frac{1}{\delta} \int_{0}^{T}|f(t)|^{2} e^{-\delta t} \mathrm{~d} t+\frac{\varepsilon K}{\delta}\right) .
\end{aligned}
$$

This proves (4.14). 
Now for getting (4.15) we proceed as above and taking supremum before taking the expectaion, in (4.17)

$$
\begin{aligned}
& \mathbb{E}\left[\sup _{0 \leq t \leq T}\left|u^{n, \varepsilon}(t)\right|^{2} e^{-\delta t}\right]+2 \nu \int_{0}^{T} \mathbb{E}\left\|u^{n, \varepsilon}(t)\right\|^{2} e^{-\delta t} \mathrm{~d} t \\
& \leq \mathbb{E}|u(0)|^{2}+\frac{1}{\delta} \int_{0}^{T}|f(t)|^{2} e^{-\delta t} \mathrm{~d} t+\mathbb{E} \sup _{0 \leq s \leq T} \int_{0}^{s} \varepsilon \mid \sigma_{n}\left(t,\left.u^{n, \varepsilon}(t)\right|^{2} e^{-\delta t} \mathrm{~d} t\right. \\
&+2 \sqrt{\varepsilon} \mathbb{E} \sup _{0 \leq s \leq T} \int_{0}^{s}\left(\sigma_{n}\left(t, u^{n, \varepsilon}(t)\right), u^{n, \varepsilon}(t)\right) e^{-\delta t} \mathrm{~d} W_{n}(t) \\
&+\mathbb{E} \sup _{0 \leq s \leq T} \int_{0}^{s} e^{-\delta t} \int_{Z} \varepsilon\left|g^{n}\left(u^{n, \varepsilon}(t-), z\right)\right|^{2} \lambda(d z) \mathrm{d} t \\
&+2 \mathbb{E} \sup _{0 \leq s \leq T} \int_{0}^{s} e^{-\delta t} \int_{Z} \varepsilon\left(u^{n, \varepsilon}(t-), g^{n}\left(u^{n, \varepsilon}(t-), z\right)\right) \tilde{N}(d t, d z) \\
& \leq \mathbb{E}|u(0)|^{2}+\frac{1}{\delta} \int_{0}^{T}|f(t)|^{2} e^{-\delta t} \mathrm{~d} t+\varepsilon K \mathbb{E}\left[\int_{0}^{T} \sup _{0 \leq s \leq t}\left|u^{n, \varepsilon}(s)\right|^{2} e^{-\delta t} \mathrm{~d} t\right]+\frac{\varepsilon K}{\delta} \\
&+2 \sqrt{\varepsilon} \mathbb{E} \sup _{0 \leq s \leq T}\left|\int_{0}^{s}\left(\sigma_{n}\left(t, u^{n, \varepsilon}(t)\right), u^{n, \varepsilon}(t)\right) e^{-\delta t} \mathrm{~d} W_{n}(t)\right| \\
&+2 \varepsilon \mathbb{E} \sup _{0 \leq s \leq T}\left|\int_{0}^{s} e^{-\delta t} \int_{Z}\left(u^{n, \varepsilon}(t-), g^{n}\left(u^{n, \varepsilon}(t-), z\right)\right) \tilde{N}(d t, d z)\right| \cdot
\end{aligned}
$$

Next we consider

$$
2 \sqrt{\varepsilon} \mathbb{E}\left[\sup _{0 \leq s \leq T}\left|\int_{0}^{s}\left(\sigma_{n}\left(t, u^{n, \varepsilon}(t)\right), u^{n, \varepsilon}(t)\right) e^{-\delta t} \mathrm{~d} W_{n}(t)\right|\right]
$$

and applying Burkholder-Davis-Gundy Inequality, Young's Inequality and condition (H.2), we get

$$
\begin{aligned}
& 2 \sqrt{\varepsilon} \mathbb{E}\left[\sup _{0 \leq s \leq T}\left|\int_{0}^{s}\left(\sigma_{n}\left(t, u^{n, \varepsilon}(t)\right), u^{n, \varepsilon}(t)\right) e^{-\delta t} \mathrm{~d} W_{n}(t)\right|\right] \\
& \quad \leq 2 \sqrt{2 \varepsilon} \mathbb{E}\left[\int_{0}^{T} \mid \sigma_{n}\left(t,\left.u^{n, \varepsilon}(t)\right|^{2}\left|u^{n, \varepsilon}(t)\right|^{2} e^{-2 \delta t} \mathrm{~d} t\right]^{1 / 2}\right. \\
& \quad \leq 2 \sqrt{2 \varepsilon K} \mathbb{E}\left[\int_{0}^{T}\left(1+\left|u^{n, \varepsilon}(t)\right|^{2}\right)\left|u^{n, \varepsilon}(t)\right|^{2} e^{-2 \delta t} \mathrm{~d} t\right]^{1 / 2} \\
& \quad \leq 2 \sqrt{2 \varepsilon K} \mathbb{E}\left[\left(\sup _{0 \leq t \leq T}\left|u^{n, \varepsilon}(t)\right| e^{-\delta t / 2}\right)\left(\int_{0}^{T}\left(1+\left|u^{n, \varepsilon}(t)\right|^{2}\right) e^{-\delta t} \mathrm{~d} t\right)^{1 / 2}\right] \\
& \quad \leq \frac{1}{4} \mathbb{E}\left[\sup _{0 \leq t \leq T}\left|u^{n, \varepsilon}(t)\right|^{2} e^{-\delta t}\right]+8 \varepsilon K \mathbb{E} \int_{0}^{T}\left|u^{n, \varepsilon}(t)\right|^{2} e^{-\delta t} \mathrm{~d} t+\frac{8 \varepsilon K}{\delta} \\
& \quad \leq \frac{1}{4} \mathbb{E}\left[\sup _{0 \leq t \leq T}\left|u^{n, \varepsilon}(t)\right|^{2} e^{-\delta t}\right]+8 \varepsilon K \mathbb{E} \int_{0}^{T} \sup _{0 \leq s \leq t}\left|u^{n, \varepsilon}(s)\right|^{2} e^{-\delta t} \mathrm{~d} t+\frac{8 \varepsilon K}{\delta} .
\end{aligned}
$$


Now again applying Burkholder-Davis-Gundy Inequality in the form given in Lemma 2.27, Young's Inequality and (H.2) to the term

$$
2 \varepsilon \mathbb{E}\left[\sup _{0 \leq s \leq T}\left|\int_{0}^{s} e^{-\delta t} \int_{Z}\left(u^{n, \varepsilon}(t-), g^{n}\left(u^{n, \varepsilon}(t-), z\right)\right) \tilde{N}(d t, d z)\right|\right]
$$

we get,

$$
\begin{aligned}
2 \varepsilon \mathbb{E} & \left.\sup _{0 \leq s \leq T}\left|\int_{0}^{s} e^{-\delta t} \int_{Z}\left(u^{n, \varepsilon}(t), g^{n}\left(u^{n, \varepsilon}(t-), z\right)\right) \tilde{N}(d t, d z)\right|\right] \\
& \leq 2 \sqrt{2} \varepsilon \mathbb{E}\left(\int_{0}^{T} \int_{Z}\left|\left(u^{n, \varepsilon}(t), g^{n}\left(u^{n, \varepsilon}(t), z\right)\right) e^{-\delta t}\right|^{2} \lambda(d z) d t\right)^{1 / 2} \\
& \leq 2 \sqrt{2} \varepsilon \mathbb{E}\left(\int_{0}^{T} \int_{Z}\left|g^{n}\left(u^{n, \varepsilon}(t), z\right)\right|^{2}\left|u^{n, \varepsilon}(t)\right|^{2} e^{-2 \delta t} \lambda(d z) \mathrm{d} t\right)^{1 / 2} \\
& \leq 2 \sqrt{2} \varepsilon \mathbb{E}\left(\int_{0}^{T} K\left(1+\left|u^{n, \varepsilon}(t)\right|^{2}\right)\left|u^{n, \varepsilon}(t)\right|^{2} e^{-2 \delta t} \lambda(d z) \mathrm{d} t\right)^{1 / 2} \\
& \leq 2 \sqrt{2} \varepsilon \mathbb{E}\left[\left(\sup _{0 \leq t \leq T}\left|u^{n, \varepsilon}(t)\right| e^{-\delta t / 2}\right)\left(\int_{0}^{T} K\left(1+\left|u^{n, \varepsilon}(t)\right|^{2}\right) e^{-\delta t} \mathrm{~d} t\right)^{1 / 2}\right] \\
& \leq \frac{1}{4} \mathbb{E}\left[\sup _{0 \leq t \leq T}\left|u^{n, \varepsilon}(t)\right|^{2} e^{-\delta t}\right]+8 \varepsilon^{2} K \mathbb{E} \int_{0}^{T} \sup _{0 \leq s \leq t}\left|u^{n, \varepsilon}(s)\right|^{2} e^{-\delta t} \mathrm{~d} t+\frac{8 \varepsilon^{2} K}{\delta} .
\end{aligned}
$$

By applying (4.21) and (4.22) in (4.20) one can deduce that

$$
\begin{aligned}
\mathbb{E}\left[\sup _{0 \leq t \leq T}\left|u^{n, \varepsilon}(t)\right|^{2} e^{-\delta t}\right]+4 \nu \int_{0}^{T} \mathbb{E}\left\|u^{n, \varepsilon}(t)\right\|^{2} e^{-\delta t} \mathrm{~d} t \\
\leq 2 \mathbb{E}|u(0)|^{2}+\frac{2}{\delta} \int_{0}^{T}|f(t)|^{2} e^{-\delta t} \mathrm{~d} t+\frac{2 \varepsilon K(9+8 \varepsilon)}{\delta} \\
\quad+2 \varepsilon K(9+8 \varepsilon) \mathbb{E} \int_{0}^{T} \sup _{0 \leq s \leq t}\left|u^{n, \varepsilon}(s)\right|^{2} e^{-\delta t} \mathrm{~d} t .
\end{aligned}
$$

From the above expression one can write,

$$
\begin{aligned}
\mathbb{E}\left[\sup _{0 \leq t \leq T}\left|u^{n, \varepsilon}(t)\right|^{2} e^{-\delta t}\right] \leq & 2 \mathbb{E}|u(0)|^{2}+\frac{2}{\delta} \int_{0}^{T}|f(t)|^{2} e^{-\delta t} \mathrm{~d} t \\
& +\frac{2 \varepsilon K(9+8 \varepsilon)}{\delta} \\
& +2 \varepsilon K(9+8 \varepsilon) \mathbb{E} \int_{0}^{T} \sup _{0 \leq s \leq t}\left|u^{n, \varepsilon}(s)\right|^{2} e^{-\delta t} \mathrm{~d} t .
\end{aligned}
$$


Applying Gronwall's Inequality

$$
\begin{aligned}
& \mathbb{E}\left[\sup _{0 \leq t \leq T}\left|u^{n, \varepsilon}(t)\right|^{2} e^{-\delta t}\right] \\
& \leq e^{2 \varepsilon K T(9+8 \varepsilon)}\left[2 \mathbb{E}|u(0)|^{2}+\frac{2}{\delta} \int_{0}^{T}|f(t)|^{2} e^{-\delta t} \mathrm{~d} t+\frac{2 \varepsilon K(9+8 \varepsilon)}{\delta}\right] .
\end{aligned}
$$

Using (4.24) in (4.23) we get

$$
\begin{aligned}
\mathbb{E} & \left.\sup _{0 \leq t \leq T}\left|u^{n, \varepsilon}(t)\right|^{2} e^{-\delta t}\right]+4 \nu \int_{0}^{T} \mathbb{E}\left\|u^{n, \varepsilon}(t)\right\|^{2} e^{-\delta t} \mathrm{~d} t \\
\leq & \left(1+2 \varepsilon K T(9+8 \varepsilon) e^{2 \varepsilon K T(9+8 \varepsilon)}\right) \\
& \times\left(2 \mathbb{E}|u(0)|^{2}+\frac{2}{\delta} \int_{0}^{T}|f(t)|^{2} e^{-\delta t} \mathrm{~d} t+\frac{2 \varepsilon K(9+8 \varepsilon)}{\delta}\right) .
\end{aligned}
$$

By using the above we get the required result (4.15)

Definition 4.3. (Strong Solution) A strong solution $u^{\varepsilon}$ is defined on a given probability space $\left(\Omega, \mathcal{F}, \mathcal{F}_{t}, P\right)$ as a $\mathrm{L}^{2}\left(\Omega ; \mathrm{L}^{\infty}(0, T ; H) \cap \mathrm{L}^{2}(0, T ; V) \cap\right.$ $\mathcal{D}(0, T ; H))$ valued adapted process which satisfies the stochastic GOY model

$$
\begin{aligned}
\mathrm{d} u^{\varepsilon}+ & {\left[\nu A u^{\varepsilon}+B\left(u^{\varepsilon}, u^{\varepsilon}\right)\right] \mathrm{d} t=f(t) \mathrm{d} t+\sqrt{\varepsilon} \sigma\left(t, u^{\varepsilon}\right) \mathrm{d} W(t) } \\
+\varepsilon \int_{Z} g\left(u^{\varepsilon}, z\right) \tilde{N}(d t, d z) & \\
u^{\varepsilon}(0) & =u_{0}
\end{aligned}
$$

in the weak sense and also the energy inequalities in Theorems 4.1 and 4.2 .

Theorem 4.4. Let $u(0)$ be $\mathcal{F}_{0}$ measurable and $\mathbb{E}\left|u_{0}\right|^{2}<\infty$. Let $f \in$ $\mathrm{L}^{2}\left(0, T ; V^{\prime}\right)$. We also assume that $0<\varepsilon<\frac{\nu}{C}$ and the diffusion coefficient satisfies the conditions (H.1)-(H.3). Then there exists unique adapted process $u^{\varepsilon}(t, x, w)$ with the regularity

$$
u^{\varepsilon} \in \mathrm{L}^{2}\left(\Omega ; \mathcal{D}(0, T ; H) \cap \mathrm{L}^{2}(0, T ; V)\right)
$$

satisfying the stochastic GOY model (4.26) and the a priori bounds in Theorems 4.1 and 4.2 . 


\section{Proof. Part I (Existence)}

Using the a priori estimate in the Theorems 4.1 and 4.2 , it follows from the Banach-Alaoglu theorem that along a subsequence, the Galerkin approximations $\left\{u^{n, \varepsilon}\right\}$ have the following limits:

$$
\begin{aligned}
& u^{n, \varepsilon} \longrightarrow u^{\varepsilon} \quad \text { weak star in } \mathrm{L}^{2}\left(\Omega ; \mathrm{L}^{\infty}(0, T ; H)\right) \cap \mathrm{L}^{2}\left(\Omega ; \mathrm{L}^{2}(0, T ; V)\right), \\
& F\left(u^{n, \varepsilon}\right) \longrightarrow F_{0}^{\varepsilon} \quad \text { weakly in } \mathrm{L}^{2}\left(\Omega ; \mathrm{L}^{2}\left(0, T ; V^{\prime}\right)\right), \\
& \sigma_{n}\left(\cdot, u^{n, \varepsilon}\right) \longrightarrow S^{\varepsilon} \quad \text { weakly in } \mathrm{L}^{2}\left(\Omega ; \mathrm{L}^{2}\left(0, T ; \mathrm{L}_{Q}\right)\right) \\
& g^{n}\left(u^{n, \varepsilon}, \cdot\right) \longrightarrow G^{\varepsilon} \quad \text { weakly in } \mathbb{H}_{\lambda}^{2}([0, T] \times Z ; H) .
\end{aligned}
$$

The assertion of the second statement holds since $F\left(u^{n, \varepsilon}\right)$ is bounded in $\mathrm{L}^{2}\left(\Omega ; \mathrm{L}^{2}\left(0, T ; V^{\prime}\right)\right)$. Likewise since diffusion coefficient has the linear growth property and $u^{n, \varepsilon}$ is bounded in $\mathrm{L}^{2}(0, T ; V)$ uniformly in $n$, the last two statements hold. Then $u^{\varepsilon}$ has the Itô differential

$$
\begin{gathered}
\mathrm{d} u^{\varepsilon}(t)=F_{0}^{\varepsilon}(t) \mathrm{d} t+\sqrt{\varepsilon} S^{\varepsilon}(t) \mathrm{d} W(t)+\varepsilon \int_{Z} G^{\varepsilon}(t) \tilde{N}(d t, d z) \\
\text { weakly in } \mathrm{L}^{2}\left(\Omega ; \mathrm{L}^{2}\left(0, T ; V^{\prime}\right)\right) .
\end{gathered}
$$

Let us set,

$$
r(t):=\frac{2}{\nu^{3}} \int_{0}^{t}\left\|v^{\varepsilon}(s)\right\|_{\mathrm{L}^{4}}^{4} \mathrm{~d} s,
$$

where $v^{\varepsilon}(t, x, \omega)$ is any adapted process in $\mathrm{L}^{\infty}(\Omega \times(0, T) ; H)$. Here we suppress the dependence of $\varepsilon$ in the notation of $r$ to make it easier to read. Then applying the Itô Lemma to the function $2 e^{-r(t)}\left|u^{n, \varepsilon}(t)\right|^{2}$, one obtains

$$
\begin{aligned}
\mathrm{d}\left[e^{-r(t)}\left|u^{n, \varepsilon}(t)\right|^{2}\right]= & e^{-r(t)}\left(2 F\left(u^{n, \varepsilon}(t)\right)-\dot{r}(t) u^{n, \varepsilon}(t), u^{n, \varepsilon}(t)\right) \mathrm{d} t \\
& +\varepsilon e^{-r(t)}\left|\sigma_{n}\left(t, u^{n, \varepsilon}(t)\right)\right|_{\mathrm{L}_{Q}}^{2} \mathrm{~d} t \\
& +2 \sqrt{\varepsilon} e^{-r(t)}\left(\sigma_{n}\left(t, u^{n, \varepsilon}(t)\right), u^{n, \varepsilon}(t)\right) \mathrm{d} W(t) \\
& +e^{-r(t)} \epsilon \int_{Z}\left|g^{n}\left(u^{n, \varepsilon}(t-), z\right)\right|^{2} N(d t, d z) \\
& +2 e^{-r(t)} \varepsilon \int_{Z}\left(u^{n, \varepsilon}(t-), g^{n}\left(u^{n, \varepsilon}(t-), z\right)\right) \tilde{N}(d t, d z) .
\end{aligned}
$$

Integrating between $0 \leq t \leq T$ and taking expectation,

$$
\begin{aligned}
\mathbb{E}[ & \left.e^{-r(T)}\left|u^{n, \varepsilon}(T)\right|^{2}-\left|u^{n, \varepsilon}(0)\right|^{2}\right] \\
= & \mathbb{E}\left[\int_{0}^{T} e^{-r(t)}\left(2 F\left(u^{n, \varepsilon}(t)\right)-\dot{r}(t) u^{n, \varepsilon}(t), u^{n, \varepsilon}(t)\right) \mathrm{d} t\right] \\
& +\mathbb{E} \int_{0}^{T} e^{-r(t)} \varepsilon\left|\sigma_{n}\left(t, u^{n, \varepsilon}(t)\right)\right|_{\mathrm{L}_{Q}}^{2} \mathrm{~d} t \\
& +2 \sqrt{\varepsilon} \mathbb{E} \int_{0}^{T} e^{-r(t)}\left(\sigma_{n}\left(t, u^{n, \varepsilon}(t)\right), u^{n, \varepsilon}(t)\right) \mathrm{d} W(t)
\end{aligned}
$$


Vol. 18 (2011)

$$
\begin{aligned}
& +\mathbb{E} \varepsilon \int_{0}^{T} e^{-r(t)} \int_{Z}\left|g^{n}\left(u^{n, \varepsilon}(t), z\right)\right|^{2} \lambda(d z) \mathrm{d} t \\
& +2 \mathbb{E} \int_{0}^{T} e^{-r(t)} \varepsilon \int_{Z}\left(u^{n, \varepsilon}(t-), g^{n}\left(u^{n, \varepsilon}(t-), z\right)\right) \tilde{N}(d t, d z) .
\end{aligned}
$$

But the terms

$$
2 \sqrt{\varepsilon} \int_{0}^{T} e^{-r(t)}\left(\sigma_{n}\left(t, u^{n, \varepsilon}(t)\right), u^{n, \varepsilon}(t)\right) \mathrm{d} W(t)
$$

and

$$
2 \int_{0}^{T} e^{-r(t)} \varepsilon \int_{Z}\left(u^{n, \varepsilon}(t-), g^{n}\left(u^{n, \varepsilon}(t-), z\right)\right) \tilde{N}(d t, d z)
$$

are martingales and having zero averages. Hence we get

$$
\begin{aligned}
\mathbb{E} & {\left[e^{-r(T)}\left|u^{n, \varepsilon}(T)\right|^{2}-\left|u^{n, \varepsilon}(0)\right|^{2}\right] } \\
= & \mathbb{E}\left[\int_{0}^{T} e^{-r(t)}\left(2 F\left(u^{n, \varepsilon}(t)\right)-\dot{r}(t) u^{n, \varepsilon}(t), u^{n, \varepsilon}(t)\right) \mathrm{d} t\right] \\
& +\mathbb{E} \int_{0}^{T} e^{-r(t)} \varepsilon\left|\sigma_{n}\left(t, u^{n, \varepsilon}(t)\right)\right|_{\mathrm{L}_{Q}}^{2} \mathrm{~d} t \\
& +\mathbb{E} \int_{0}^{T} e^{-r(t)} \varepsilon \int_{Z}\left|g^{n}\left(u^{n, \varepsilon}(t), z\right)\right|^{2} \lambda(d z) \mathrm{d} t
\end{aligned}
$$

Then by the lower semi-continuity property of the weak convergence,

$$
\begin{array}{rl}
\liminf _{n} & \mathbb{E}\left[\int_{0}^{T} e^{-r(t)}\left(2 F\left(u^{n, \varepsilon}(t)\right)-\dot{r}(t) u^{n, \varepsilon}(t), u^{n, \varepsilon}(t)\right) \mathrm{d} t\right. \\
& \left.+\int_{0}^{T} e^{-r(t)} \varepsilon\left|\sigma_{n}\left(t, u^{n, \varepsilon}(t)\right)\right|_{\mathrm{L}_{Q}}^{2} \mathrm{~d} t+\int_{0}^{T} e^{-r(t)} \varepsilon \int_{Z}\left|g^{n}\left(u^{n, \varepsilon}(t), z\right)\right|^{2} \lambda(d z) \mathrm{d} t\right] \\
= & \lim _{n} \inf \mathbb{E}\left[e^{-r(T)}\left|u^{n, \varepsilon}(T)\right|^{2}-\left|u^{n, \varepsilon}(0)\right|^{2}\right] \\
\geq & \mathbb{E}\left[e^{-r(T)}\left|u^{\varepsilon}(T)\right|^{2}-\left|u^{\varepsilon}(0)\right|^{2}\right] \\
= & \mathbb{E}\left[\int_{0}^{T} e^{-r(t)}\left(2 F_{0}^{\varepsilon}(t)-\dot{r}(t) u^{\varepsilon}(t), u^{\varepsilon}(t)\right) \mathrm{d} t+\varepsilon \int_{0}^{T} e^{-r(t)}\left|S^{\varepsilon}\right|_{\mathrm{L}_{Q}}^{2} \mathrm{~d} t\right. \\
\left.\quad+\int_{0}^{T} e^{-r(t)} \varepsilon \int_{Z}\left|G^{\varepsilon}\right|^{2} \lambda(d z) \mathrm{d} t\right]
\end{array}
$$

Hence we get

$$
\begin{array}{rl}
\liminf _{n} & \mathbb{E}\left[\int_{0}^{T} e^{-r(t)}\left(2 F\left(u^{n, \varepsilon}(t)\right)-\dot{r}(t) u^{n, \varepsilon}(t), u^{n, \varepsilon}(t)\right) \mathrm{d} t\right. \\
\left.\quad+\int_{0}^{T} e^{-r(t)} \varepsilon\left|\sigma_{n}\left(t, u^{n, \varepsilon}(t)\right)\right|_{\mathrm{L}_{Q}}^{2} \mathrm{~d} t+\int_{0}^{T} e^{-r(t)} \varepsilon \int_{Z}\left|g^{n}\left(u^{n, \varepsilon}(t), z\right)\right|^{2} \lambda(d z) \mathrm{d} t\right]
\end{array}
$$




$$
\begin{aligned}
\geq & \mathbb{E}\left[\int_{0}^{T} e^{-r(t)}\left(2 F_{0}^{\varepsilon}(t)-\dot{r}(t) u^{\varepsilon}(t), u^{\varepsilon}(t)\right) \mathrm{d} t+\varepsilon \int_{0}^{T} e^{-r(t)}\left|S^{\varepsilon}\right|_{\mathrm{L}_{Q}}^{2} \mathrm{~d} t\right. \\
& \left.+\int_{0}^{T} e^{-r(t)} \varepsilon \int_{Z}\left|G^{\varepsilon}\right|^{2} \lambda(d z) \mathrm{d} t\right] .
\end{aligned}
$$

Now by monotonicity property from Lemma 3.6,

$$
\begin{aligned}
2 \mathbb{E}\left[\int_{0}^{T} e^{-r(t)}\left(F\left(u^{n, \varepsilon}(t)\right)-F\left(v^{\varepsilon}(t)\right), u^{n, \varepsilon}(t)-v^{\varepsilon}(t)\right) \mathrm{d} t\right] \\
-\mathbb{E}\left[\int_{0}^{T} e^{-r(t)} \dot{r}(t)\left|u^{n, \varepsilon}(t)-v^{\varepsilon}(t)\right|^{2} \mathrm{~d} t\right] \\
+\mathbb{E}\left[\int_{0}^{T} e^{-r(t)} \varepsilon\left|\sigma_{n}\left(t, u^{n, \varepsilon}(t)\right)-\sigma_{n}\left(t, v^{\varepsilon}(t)\right)\right|_{\mathrm{L}_{Q}}^{2} \mathrm{~d} t\right] \\
+\mathbb{E}\left[\int_{0}^{T} e^{-r(t)} \int_{Z} \varepsilon\left|g^{n}\left(u^{n, \varepsilon}(t), z\right)-g^{n}\left(v^{\varepsilon}(t), z\right)\right|^{2} \lambda(d z) \mathrm{d} t\right] \\
\leq 0 .
\end{aligned}
$$

Rearranging the terms,

$$
\begin{aligned}
& \mathbb{E}\left[\int_{0}^{T} e^{-r(t)}\left(2 F\left(u^{n, \varepsilon}(t)\right)-\dot{r}(t) u^{n, \varepsilon}(t), u^{n, \varepsilon}(t)\right) \mathrm{d} t\right. \\
& \left.\quad+\int_{0}^{T} e^{-r(t)} \varepsilon\left|\sigma_{n}\left(t, u^{n, \varepsilon}(t)\right)\right|_{\mathrm{L}_{Q}}^{2} \mathrm{~d} t+\int_{0}^{T} e^{-r(t)} \int_{Z} \varepsilon\left|g^{n}\left(u^{n, \varepsilon}(t), z\right)\right|^{2} \lambda(d z) \mathrm{d} t\right] \\
& \leq \mathbb{E}\left[\int_{0}^{T} e^{-r(t)}\left(2 F\left(u^{n, \varepsilon}(t)\right)-\dot{r}(t)\left(2 u^{n, \varepsilon}(t)-v^{\varepsilon}(t)\right), v^{\varepsilon}(t)\right) \mathrm{d} t\right] \\
& +\mathbb{E}\left[\int_{0}^{T} e^{-r(t)}\left(2 F\left(v^{\varepsilon}(t)\right), u^{n, \varepsilon}(t)-v^{\varepsilon}(t)\right) \mathrm{d} t\right] \\
& +\varepsilon \mathbb{E}\left[\int_{0}^{T} e^{-r(t)}\left(2 \sigma_{n}\left(t, u^{n, \varepsilon}(t)\right)-\sigma_{n}\left(t, v^{\varepsilon}(t)\right), \sigma_{n}\left(t, v^{\varepsilon}(t)\right)\right)_{\mathrm{L}_{Q}} \mathrm{~d} t\right] \\
& \quad+\varepsilon \mathbb{E}\left[\int_{0}^{T} e^{-r(t)} \int_{Z}\left(2 g^{n}\left(u^{n, \varepsilon}(t), z\right)-g^{n}\left(v^{\varepsilon}(t), z\right), g^{n}\left(v^{\varepsilon}(t), z\right)\right) \lambda(d z) \mathrm{d} t\right] .
\end{aligned}
$$

Taking limit in $n$, using the result from (4.29), we get

$$
\begin{aligned}
& \mathbb{E}\left[\int_{0}^{T} e^{-r(t)}\left(2 F_{0}^{\varepsilon}(t)-\dot{r}(t) u^{\varepsilon}(t), u^{\varepsilon}(t)\right) \mathrm{d} t+\varepsilon \int_{0}^{T} e^{-r(t)}\left|S^{\varepsilon}\right|_{\mathrm{L}_{Q}}^{2} \mathrm{~d} t\right. \\
& \left.\quad+\int_{0}^{T} e^{-r(t)} \int_{Z} \varepsilon\left|G^{\varepsilon}\right|^{2} \lambda(d z) \mathrm{d} t\right] \\
& \leq \mathbb{E}\left[\int_{0}^{T} e^{-r(t)}\left(2 F_{0}^{\varepsilon}(t)-\dot{r}(t)\left(2 u^{\varepsilon}(t)-v^{\varepsilon}(t)\right), v^{\varepsilon}(t)\right) \mathrm{d} t\right]
\end{aligned}
$$




$$
\begin{aligned}
& +\mathbb{E}\left[\int_{0}^{T} e^{-r(t)}\left(2 F\left(v^{\varepsilon}(t)\right), u^{\varepsilon}(t)-v^{\varepsilon}(t)\right) \mathrm{d} t\right] \\
& +\varepsilon \mathbb{E}\left[\int_{0}^{T} e^{-r(t)}\left(2 S^{\varepsilon}(t)-\sigma\left(t, v^{\varepsilon}(t)\right), \sigma\left(t, v^{\varepsilon}(t)\right)\right)_{\mathrm{L}_{Q}} \mathrm{~d} t\right] \\
& +\varepsilon \mathbb{E}\left[\int_{0}^{T} e^{-r(t)} \int_{Z}\left(2 G^{\varepsilon}(t)-g\left(v^{\varepsilon}(t), z\right), g\left(v^{\varepsilon}(t), z\right)\right) \lambda(d z) \mathrm{d} t\right] .
\end{aligned}
$$

Rearranging the terms, we obtain

$$
\begin{aligned}
& \mathbb{E}\left[\int_{0}^{T} e^{-r(t)}\left(2 F_{0}^{\varepsilon}(t)-2 F\left(v^{\varepsilon}(t)\right), u^{\varepsilon}(t)-v^{\varepsilon}(t)\right) \mathrm{d} t\right] \\
& +\mathbb{E}\left[\int_{0}^{T} e^{-r(t)} \dot{r}(t)\left|u^{\varepsilon}(t)-v^{\varepsilon}(t)\right|^{2} \mathrm{~d} t\right] \\
& +\varepsilon \mathbb{E}\left[\int_{0}^{T} e^{-r(t)}\left\|S(t)-\sigma\left(t, v^{\varepsilon}(t)\right)\right\|_{\mathrm{L}_{Q}}^{2} \mathrm{~d} t\right] \\
& +\varepsilon \mathbb{E}\left[\int_{0}^{T} e^{-r(t)} \int_{Z}\left\|G(t)-g\left(v^{\varepsilon}(t), z\right)\right\|^{2} \lambda(d z) \mathrm{d} t\right] \\
& \leq 0 .
\end{aligned}
$$

Notice that for $v^{\varepsilon}=u^{\varepsilon}, S(t)=\sigma\left(t, u^{\varepsilon}(t)\right)$ and $G(t)=g\left(u^{\varepsilon}(t), z\right)$. Take $v^{\varepsilon}=$ $u^{\varepsilon}-\mu w^{\varepsilon}$ with $\mu>0$ and $w^{\varepsilon}$ is an adapted process in $L^{2}(\Omega ; \mathcal{D}(0, T ; H) \cap$ $\left.\mathrm{L}^{2}(0, T ; V)\right)$. Then,

$$
\begin{gathered}
\mu \mathbb{E}\left[\int_{0}^{T} e^{-r(t)}\left(2 F_{0}^{\varepsilon}(t)-2 F\left(u^{\varepsilon}-\mu w^{\varepsilon}\right)(t), w^{\varepsilon}(t)\right) \mathrm{d} t\right. \\
\left.+\mu \int_{0}^{T} e^{-r(t)} \dot{r}(t)\left|w^{\varepsilon}(t)\right|^{2} \mathrm{~d} t\right] \leq 0 .
\end{gathered}
$$

Dividing by $\mu$ on both sides of the inequality above and letting $\mu$ go to 0 , one obtains

$$
\mathbb{E}\left[\int_{0}^{T} e^{-r(t)}\left(F_{0}^{\varepsilon}(t)-F\left(u^{\varepsilon}(t)\right), w^{\varepsilon}(t)\right) \mathrm{d} t\right] \leq 0 .
$$

Since $w^{\varepsilon}$ is arbitrary, we conclude that $F_{0}^{\varepsilon}(t)=F\left(u^{\varepsilon}(t)\right)$. Thus the existence of the strong solution of the stochastic GOY model (4.26) has been proved. Part II (Uniqueness)

If $v^{\varepsilon} \in \mathrm{L}^{2}\left(\Omega ; \mathcal{D}(0, T ; H) \cap \mathrm{L}^{2}(0, T ; V)\right)$ be another solution of the Eq. (4.26) then $w^{\varepsilon}=u^{\varepsilon}-v^{\varepsilon}$ solves the stochastic differential equation in $\mathrm{L}^{2}\left(\Omega ; \mathrm{L}^{2}\left(0, T ; V^{\prime}\right)\right)$,

$$
\begin{aligned}
\mathrm{d} w^{\varepsilon}(t)= & \left(F\left(u^{\varepsilon}(t)\right)-F\left(v^{\varepsilon}(t)\right)\right) \mathrm{d} t+\sqrt{\varepsilon}\left(\sigma\left(t, u^{\varepsilon}(t)\right)-\sigma\left(t, v^{\varepsilon}(t)\right)\right) \mathrm{d} W(t) \\
& +\int_{Z}\left[g\left(u^{\varepsilon}(t-), z\right)-g\left(v^{\varepsilon}(t-), z\right)\right] \tilde{N}(d t, d z) .
\end{aligned}
$$


We denote $\sigma_{d}=\sigma\left(t, u^{\varepsilon}(t)\right)-\sigma\left(t, v^{\varepsilon}(t)\right)$ and $g_{d}=g\left(u^{\varepsilon}(t-), z\right)-g\left(v^{\varepsilon}(t-), z\right)$.

We now apply Itô Lemma to the function $2 e^{-r(t)}\left|w^{\varepsilon}(t)\right|^{2}$, we get

$$
\begin{aligned}
\mathrm{d}\left[e^{-r(t)}\left|w^{\varepsilon}(t)\right|^{2}\right]= & {\left[-e^{-r(t)} \dot{r}(t)\left|w^{\varepsilon}(t)\right|^{2}+2 e^{-r(t)}\left(F\left(u^{\varepsilon}(t)\right)-F\left(v^{\varepsilon}(t)\right), w^{\varepsilon}(t)\right)\right.} \\
& \left.+\varepsilon e^{-r(t)} \operatorname{Tr}\left(\sigma_{d} Q \sigma_{d}\right)\right] \mathrm{d} t+2 \sqrt{\varepsilon} e^{-r(t)}\left(\sigma_{d}, w^{\varepsilon}(t)\right) \mathrm{d} W(t) \\
& +e^{-r(t)} \varepsilon \int_{Z}\left|g_{d}\right|^{2} N(d t, d z)+2 e^{-r(t)} \varepsilon \int_{Z}\left(w^{\varepsilon}(t), g_{d}\right) \tilde{N}(d t, d z) .
\end{aligned}
$$

Now using the local monotonicity of the sum of the linear and nonlinear operators $A$ and $B$, e.g. Eq. (3.14),we obtain

$$
\begin{aligned}
\mathrm{d}\left[e^{-r(t)}\left|w^{\varepsilon}(t)\right|^{2}\right] & +\nu\left\|w^{\varepsilon}(t)\right\|^{2} e^{-r(t)} \mathrm{d} t \\
\leq & \varepsilon e^{-r(t)}\left|\sigma_{d}\right|^{2} \mathrm{~d} t+2 \sqrt{\varepsilon} e^{-r(t)}\left(\sigma_{d}, w^{\varepsilon}(t)\right) \mathrm{d} W(t) \\
& \quad+e^{-r(t)} \int_{Z} \varepsilon\left|g_{d}\right|^{2} N(d t, d z)+2 e^{-r(t)} \varepsilon \int_{Z}\left(w^{\varepsilon}(t), g_{d}\right) \tilde{N}(d t, d z) .
\end{aligned}
$$

Now integrating from $0 \leq t \leq T$ and taking the expectation on both sides and noting that $\varepsilon<\frac{\nu}{L}$. Also using the fact that

$$
2 \sqrt{\varepsilon} \int_{0}^{T} e^{-r(t)}\left(\sigma_{d}, w^{\varepsilon}(t)\right) \mathrm{d} W(t)
$$

and

$$
2 \int_{0}^{T} e^{-r(t)} \varepsilon \int_{Z}\left(w^{\varepsilon}(t), g_{d}\right) \tilde{N}(d t, d z)
$$

are martingales having zero averages, we get

$$
\begin{aligned}
& \mathbb{E}\left[e^{-r(t)}\left|w^{\varepsilon}(t)\right|^{2}\right]+\nu \mathbb{E} \int_{0}^{T} e^{-r(t)}\left\|w^{\varepsilon}(t)\right\|^{2} \mathrm{~d} t \\
& \quad \leq \mathbb{E}|w(0)|^{2}+\mathbb{E} \int_{0}^{T} e^{-r(t)} \varepsilon\left|\sigma_{d}\right|^{2} \mathrm{~d} t+\mathbb{E} \int_{0}^{T} e^{-r(t)} \int_{Z} \varepsilon\left|g_{d}\right|^{2} \lambda(d z) \mathrm{d} t
\end{aligned}
$$

Using condition (H.3), one can deduce that

$$
\mathbb{E}\left[e^{-r(t)}\left|w^{\varepsilon}(t)\right|^{2}\right]+(\nu-\varepsilon L) \int_{0}^{T} e^{-r(t)}\left\|w^{\varepsilon}(t)\right\|^{2} \mathrm{~d} t \leq \mathbb{E}|w(0)|^{2}
$$

Sine $\varepsilon<\frac{\nu}{L}$, we obtain P-a.s.

$$
\mathbb{E}\left[e^{-r(t)}\left|w^{\varepsilon}(t)\right|^{2}\right] \leq \mathbb{E}|w(0)|^{2},
$$

which assures the uniqueness of the strong solution.

Corollary 4.5. The existence and uniqueness of the strong solution of the stochastic GOY model

$$
\begin{aligned}
\mathrm{d} u^{\varepsilon}+ & {\left[\nu A u^{\varepsilon}+B\left(u^{\varepsilon}, u^{\varepsilon}\right)\right] \mathrm{d} t=f(t) \mathrm{d} t+\sqrt{\varepsilon} \sigma\left(t, u^{\varepsilon}\right) \mathrm{d} W(t) } \\
& +\int_{Z} g\left(u^{\varepsilon}, z\right) \tilde{N}(d t, d z) \\
& u^{\varepsilon}(0)=u_{0},
\end{aligned}
$$


can be proved similarly for the adapted process $u^{\varepsilon}(t, x, \omega)$ with the regularity

$$
u^{\varepsilon} \in \mathrm{L}^{2}\left(\Omega ; \mathcal{D}(0, T ; H) \cap \mathrm{L}^{2}(0, T ; V)\right)
$$

under the hypotheses

A.1. The function $\sigma \in C\left([0, T] \times V ; L_{Q}\left(H_{0} ; H\right)\right)$, and $g \in \mathbb{H}_{\lambda}^{2}([0, T] \times Z ; H)$.

A.2. For all $t \in(0, T)$, there exists a positive constant $K$ such that for all $u \in H$,

$$
\varepsilon|\sigma(t, u)|_{L_{Q}}^{2}+\int_{Z}|g(u, z)|_{H}^{2} \lambda(d z) \leq K\left(1+\|u\|^{2}\right) .
$$

A.3. For all $t \in(0, T)$, there exists a positive constant $L$ such that for all $u, v \in H$,

$$
\varepsilon|\sigma(t, u)-\sigma(t, v)|_{L_{Q}}^{2}+\int_{Z}|g(u, z)-g(v, z)|_{H}^{2} \lambda(d z) \leq L\|u-v\|^{2} .
$$

Corollary 4.6. Sabra shell model of turbulence is the other well accepted model in the literature, and the fundamental difference with the GOY model lies in the number of complex conjugation operators used in the nonlinear terms which are responsible for differences in the phase symmetries of the two models, and as a consequence, Sabra shell model exhibits shorter-ranged correlations than the GOY model (see [19]). The equations of motion of the stochastic Sabra shell model have the following form

$$
\begin{gathered}
\frac{\mathrm{d} u_{n}}{\mathrm{~d} t}+\nu k_{n}^{2} u_{n}+i\left(a k_{n+1} u_{n+2} u_{n+1}^{\star}+b k_{n} u_{n+1} u_{n-1}^{\star}\right. \\
\left.-c k_{n-1} u_{n-1} u_{n-2}\right)=f_{n}, \quad \text { for } n=1,2, \ldots,
\end{gathered}
$$

along with the boundary conditions

$$
u_{-1}=u_{0}=0 .
$$

One can deduce from the above equation in the continuous setting with Lévy noise as

$$
\begin{aligned}
& \mathrm{d} u^{\varepsilon}+ {\left[\nu A u^{\varepsilon}+B\left(u^{\varepsilon}, u^{\varepsilon}\right)\right] \mathrm{d} t=f(t) \mathrm{d} t+\sqrt{\varepsilon} \sigma\left(t, u^{\varepsilon}\right) \mathrm{d} W(t) } \\
&+\varepsilon \int_{Z} g\left(u^{\varepsilon}, z\right) \tilde{N}(d t, d z) \\
& u^{\varepsilon}(0)=u_{0},
\end{aligned}
$$

Under the Hypothesis 3.5, and under the same functional setting, the existence and uniqueness of the strong solution can be established in $\mathrm{L}^{2}(\Omega ; \mathcal{D}(0, T ; H) \cap$ $\left.\mathrm{L}^{2}(0, T ; V)\right)$.

\section{Acknowledgments}

M.T. Mohan would like to thank Council of Scientific and Industrial Research (CSIR) for his Junior Research Fellowship. He also would like to thank Indian Institute of Science Education and Research (IISER)-Thiruvananthapuram for providing stimulating scientific environment and resources. 


\section{References}

[1] de Acosta, A.: A general non-convex large deviation result with applications to stochastic equations. Prob. Theory Relat. Fields 118, 483-521 (2000)

[2] de Acosta, A.: Large deviations for vector valued Lévy processes. Stoch. Process. Appl. 51, 75-115 (1994)

[3] Albeverio, S., Wu, J.L., Zhang, T.S.: Parabolic SPDEs driven by Poisson white noise. Stoch. Process. Appl. 74, 21-36 (1998)

[4] Albeverio, S., Rüdiger, B., Wu, J.L.: Analytic and probabilistic aspects of Lévy processes and fields in quantum theory; Lévy processes: theory and applications. In: Barndorff-Nielsen, O., Mikosch, T., Resnick, S.I. (eds.) Birkhäuser, Basel (2001)

[5] Applebaum, D.: Lévy Processes and Stochastic Calculus. In: Cambridge Studies in Advanced Mathematics, vol. 93. Cambridge University press, Cambridge (2004)

[6] Barbato, D., Barsanti, M., Bessaih, H., Flandoli, F.: Some rigorous results on a stochastic Goy model. J. Stat. Phys. 125(3), 677-716 (2006)

[7] Bensoussan, A., Temam, R.: Equations aux dérivées partielles stochastiques non linéaries(1). Isr. J. Math. 11(1), 95-129 (1972)

[8] Brzeźniak, Z., Hausenblas, E.: Maximal regularity for stochastic convolutions driven by Lévy processes. Probab. Theory Relat. Fields 145, 615-637 (2009)

[9] Constantin, P., Levant, B., Titi, E.S.: Analytic study of shell models of turbulence. Phys. D 219(2), 120-141 (2006)

[10] Da Prato, G., Zabczyk, J.: Stochastic Equations in Infinite Dimensions. Cambridge University Press, Cambridge (1992)

[11] Fournier, N.: Malliavin calculus for parabolic SPDEs with jumps. Stoch. Process. Appl. 87(1), 115-147 (2000)

[12] Frisch, U.: Turbulence. Cambridge University Press, Cambridge (1995)

[13] Hausenblas, E.: Existence, uniqueness and regularity of parabolic SPDEs driven by Poisson random measure. Electron. J. Probab. 10, 1496-1546 (2005)

[14] Hausenblas, E.: Burkholder-Davis-Gundy type inequalities of the Itô stochastic integral with respect to Lévy noise on Banach spaces (2009). arxiv.org/pdf/0902.2114v3 (preprint)

[15] Ikeda, N., Watanabe, S.: Stochastic differential equations and diffusion processes. North-Holland/Kodansha, Amsterdam (1989)

[16] Kadanoff, L., Lohse, D., Wang, J., Benzi, R.: Scaling and dissipation in the GOY shell model. Phys. Fluids 7(3), 617-629 (1995)

[17] Karatzas, I., Shreve, S.: Brownian Motion and Stochastic Calculus, 2nd edn. Springer, New York (1991) 
[18] Ladyzhenskaya, O.A.: The Mathematical Theory of Viscous Incompressible Flow. Gordon and Breach, New York (1969)

[19] L'vov, V.S., Podivilov, E., Pomyalov, A., Procaccia, I., Vandembroucq, D.: Improved shell model of turbulence. Phys. Rev. E (3) 58(2), 1811-1822 (1998)

[20] Mandrekar, V., Rüdiger, B.: Existence and uniqueness of pathwise solutions for stochastic integral equations driven by Lévy noise on separable Banach spaces. Stochastics 78(4), 189-212 (2006)

[21] Manna, U., Sritharan, S.S., Sundar, P.: Large deviations for the stochastic shell model of turbulence. Nonlinear Differ. Equ. Appl. (NoDEA) 16, 493-521 (2009)

[22] Menaldi, J.L., Sritharan, S.S.: Stochastic 2-D Navier-Stokes equation. Appl. Math. Optim. 46, 31-53 (2002)

[23] Metivier, M.: Stochastic Partial Differential Equations in Infinite Dimensional Spaces. Quaderni, Scuola Normale Superiore, Pisa (1988)

[24] Mueller, C.: The heat equation with Lévy noise. Stoch. Process. Appl. 74(1), 67-82 (1998)

[25] Ohkitani, K., Yamada, M.: Temporal intermittency in the energy cascade process and local Lyapunov analysis in fully developed model of turbulence. Prog. Theor. Phys. 89, 329-341 (1989)

[26] Peszat, S., Zabczyk, J.: Stochastic partial differential equations with Lévy noise. In: Encyclopedia of Mathematics and Its Applications, vol. 113 (2007) Cambridge University Press, Cambridge

[27] Röckner, M., Zhang, T.S.: Stochastic Evolution Equations of Jump Type: Existence, Uniqueness and Large Deviation Principles. Potential Analysis 26, 255-279 (2007)

[28] Rüdiger, B.: Stochastic integration with respect to compensated Piosson randon measures on separable Banach spaces. Stoch. Stoch. Rep. 76(3), 213-242 (2004)

[29] Shlesinger, M.F., Zavslavsky, G.M., Feisch, U. (eds.): Lévy Flights and Related Topics in Physics. Springer, Berlin (1995)

[30] Temam, R.: Navier-Stokes Equations, Theory and Numerical Analysis. NorthHolland, Amsterdam (1984)

[31] Vishik, M.J., Fursikov, A.V.: Mathematical Problems of Statistical Hydromechanics. Kluwer Academic Press, Boston (1980)

[32] West, B.J.: An Essay of the Importance Being Non Linear. In: Lecture Notes in Biomathematics, vol. 62. Springer, Berlin (1985)

[33] Zhao, D., Chao, X.Y.: Global solutions of stochastic 2D Navier-Stokes equations with Lévy noise. Sci. China Ser. A 52(7), 1497-1524 (2009) 
Utpal Manna

School of Mathematics,

Indian Institute of Science Education and Research (IISER) Thiruvananthapuram, Thiruvananthapuram 695016,

Kerala, India

e-mail: manna.utpal@iisertvm.ac.in

Manil T. Mohan

School of Mathematics,

Indian Institute of Science Education and Research (IISER) Thiruvananthapuram, Thiruvananthapuram 695016,

Kerala, India

e-mail: manil@iisertvm.ac.in

Received: 3 September 2010.

Accepted: 25 February 2011. 